\title{
A RAPID PROTOTYPING METHOD FOR CONSTRUCTING A COMPLEX THREE-DIMENSIONAL SUBSTRATE
}

\author{
A Thesis \\ Presented to the Faculty of \\ California Polytechnic State University \\ San Luis Obispo, California \\ In Partial Fulfillment \\ of the Requirements for the Degree \\ Master of Science in Engineering
}

by

Kathryn Jacoba Hart

November 2009 
(C) 2009

Kathryn Jacoba Hart

ALL RIGHTS RESERVED 


\section{COMMITTEE MEMBERSHIP}

TITLE:

A rapid prototyping method for constructing a complex

three-dimensional substrate

AUTHOR: $\quad$ Kathryn Jacoba Hart

DATE SUBMITTED: $\quad$ November 2009

COMMITTEE CHAIR: $\quad$ Dr. Robert Crockett, Director, General Engineering

COMMITTEE MEMBER: Dr. Daniel Walsh, Associate Dean, College of Engineering

COMMITTEE MEMBER: Dr. Lanny Griffin, Chair, Biomedical \& General Engineering 


\begin{abstract}
A Rapid Prototyping Method for Constructing a Complex Three-Dimensional Substrate
\end{abstract}

by

Kathryn Jacoba Hart

Cell culturing on three-dimensional structures has increased the possibilities in tissue engineering and bioreactor research. These structures enable cells to differentiate, proliferate, mobilize, and function in a conformation that more accurately mimics in vivo conditions. Computer generated models aid in development and rapid alteration of three-dimensional cell substrates, defining their internal structure as well as their external morphology. The rapid transition from substrate design to a viable culture is imperative to quickly advance research in biomedical and tissue engineering applications.

The aim of this thesis is to investigate the feasibility of a rapid prototyping process by selectively cross-linking and assembling biocompatible films. This investigation revealed that selectively cross-linking and layering gelatin films could produce a three-dimensional substrate with a defined structure after dissolving uncross-linked gelatin. The study also revealed that freeze-drying aided in the rapid dissolution of uncross-linked gelatin. The line width resolution obtained during tests was $.5 \mathrm{~mm}$ using a template treatment method and was limited by the template construction resolution. Finally, alteration in treatment time, rinsing agitation, and rinsing temperature yielded stable films that better retained their size and shape compared to films produced in previous processes. 


\section{Acknowledgements}

I would like to thank Dr. Crockett, Dr. Walsh, and Dr. Griffin for helping me through this program. Dr. Walsh, thank you for kindly accepting me into the program and convincing me to take my education one step further. I appreciate your genuine concern for students and your pleasant, unassuming demeanor. Dr. Griffin, thank you for making your classes so challenging while easing the pain with a great sense of humor. I've gained a great sense of accomplishment from each of your classes. Dr. Crockett, thank you for exhibiting such enthusiasm for this project. You have overseen so many projects and worn many hats here at Cal Poly, yet somehow retained the energy to help keep me going throughout this process.

My family has been extremely supportive throughout my college career. They have made my education possible while providing emotional support during stressful times. They've also helped me to appreciate the power of a positive perspective. Mom and Lea, I especially appreciate the time you've spent helping me to talk through difficult decisions. Luke, thanks for doing so well in school. You're probably going to be supporting us all someday! Dad, thanks for the prayers. Noni and Gramps, thank you for encouraging my education and making the trip up to SLO to watch me graduate. I love you very much.

My closest friends also deserve to be mentioned here. Christina, Sean, Dan, and Heidi, thank you all. Obtaining a degree was difficult. Your distractions made it even more difficult. However, I will still mention you since you probably kept me sane through the whole process. Thank you for the fun times! 


\section{TABLE OF CONTENTS}

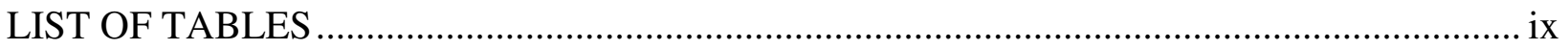

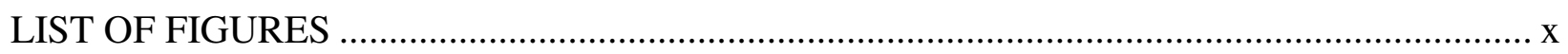

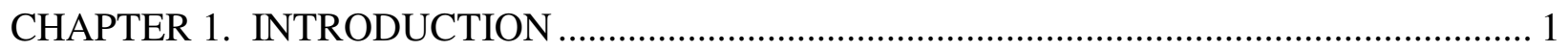

1.1. Need for the Study ......................................................................................... 1

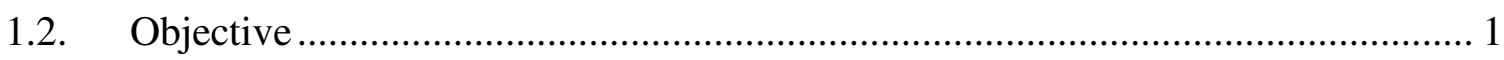

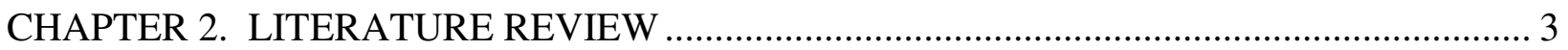

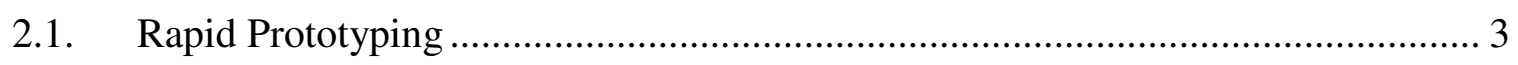

2.1.1. RP Methods.................................................................................... 3

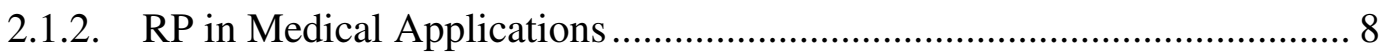

2.1.2. RP in Tissue Engineering ................................................................. 9

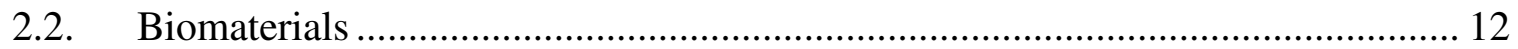

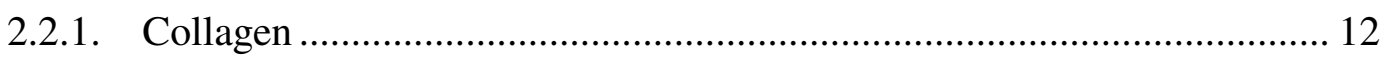

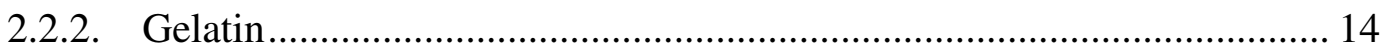

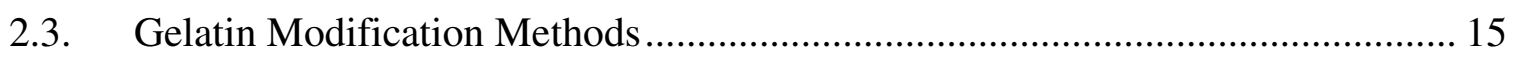

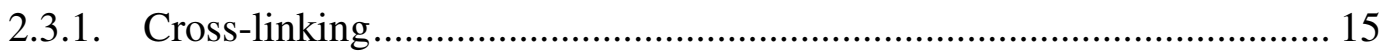

2.3.1.1. Glutaraldehyde ................................................................... 16

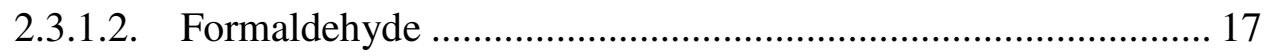

2.3.1.3. Carbodiimide.......................................................................... 18

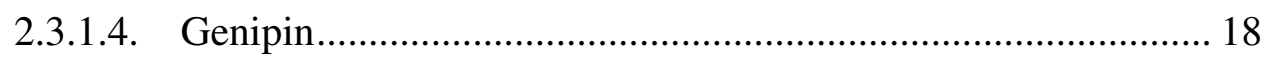

2.3.2. Gelatin Composites ......................................................................... 18

2.3.3. Physical Treatment............................................................................... 19

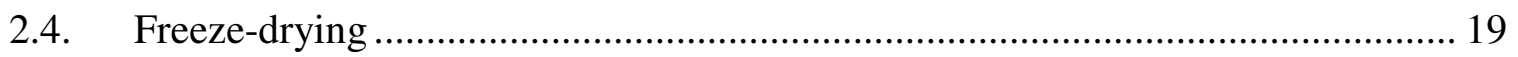




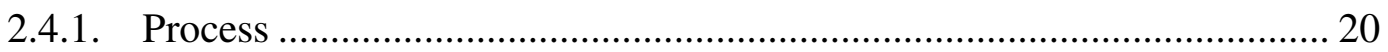

2.4.2. Considerations................................................................................. 20

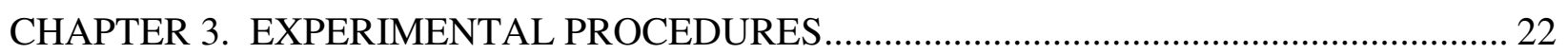

3.1. Outline Selective Treatment Experiment................................................................... 22

3.1.1. Materials and Equipment ………………………............................ 22

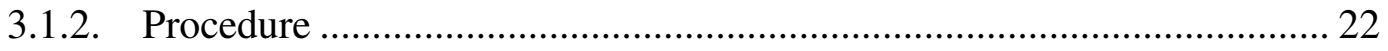

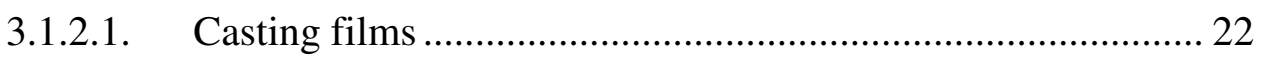

3.1.2.2. Treatment Protocol 1.............................................................. 23

3.1.2.3. Treatment Protocol 2........................................................ 24

3.2. Film Dissolution Experiment..................................................................... 25

3.2.1. Materials and Equipment ……………………................................. 25

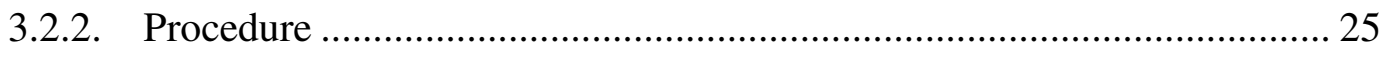

3.3. Treating Freeze-dried and Unfreeze-dried Films................................................... 26

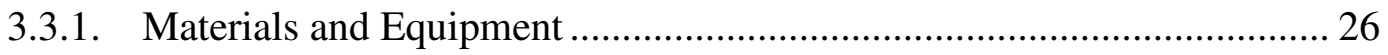

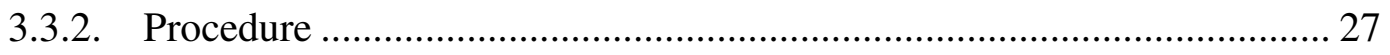

3.4. Line Width Determination Experiment ……………….................................... 28

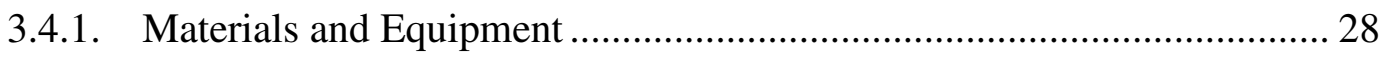

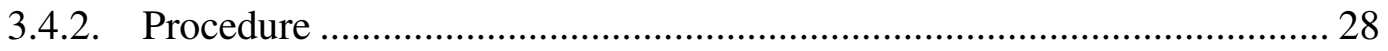

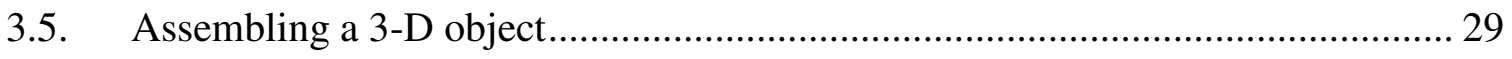

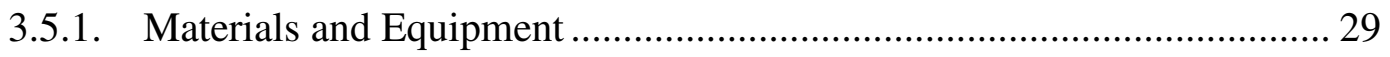

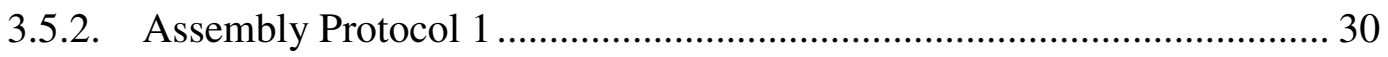

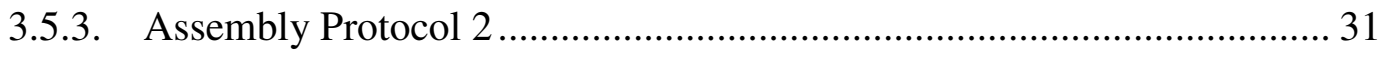

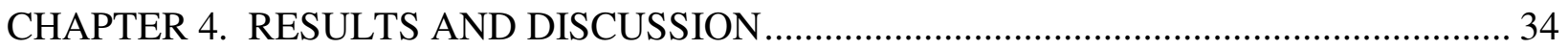


4.1. Outline Selective Treatment Experiment Results ......................................... 34

4.1.1. Results of Treatment Protocol 1 ............................................................. 34

4.1.2. Results of Treatment Protocol 2 ........................................................ 36

4.2. Results of Film Dissolution Experiment.......................................................... 37

4.3. Results of Treating Freeze-dried and Unfreeze-dried Films............................. 38

4.4. Results of the Line Width Determination Experiment ........................................ 39

4.5. Results of Assembling a 3-D Object......................................................... 40

4.5.1. Results of Assembly Protocol 1 ......................................................... 40

4.5.2. Results of Assembly Protocol 2 ....................................................... 41

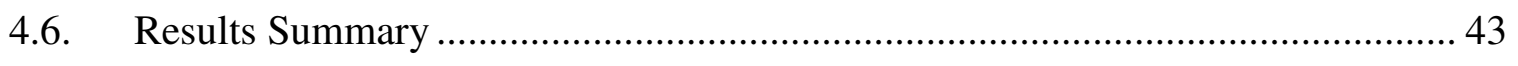

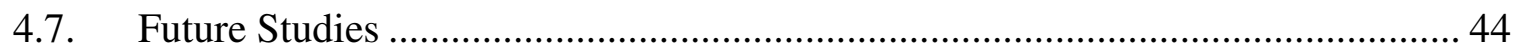

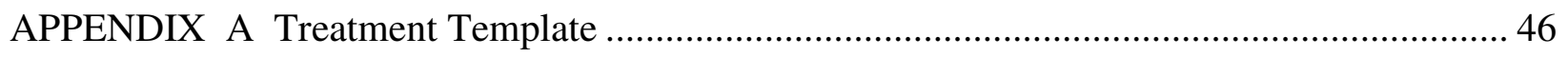

REFERENCES............................................................47 


\section{LIST OF TABLES}

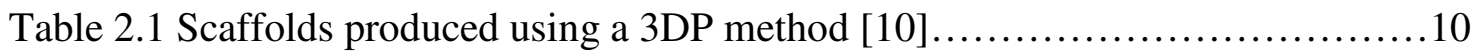

Table 3.1 Dissolution Test - Film Type vs. Time Submerged in Water...............38 


\section{LIST OF FIGURES}

Figure 2. 1 Schematic diagram of the SLA process [2] .............................................. 4

Figure 2. 2 Schematic of a Solid Ground Curing process [5] f....................................... 5

Figure 2. 3 Schematic of an LOM process [5] ........................................................ 6

Figure 2. 4 Schematic of the FDM process [5] ........................................................ 7

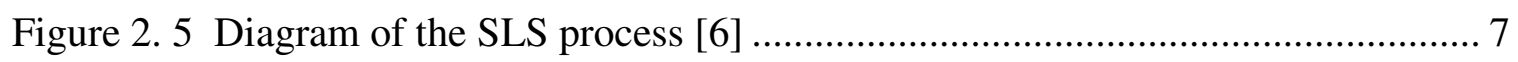

Figure 2. 6 3-Dimensional Printing Schematic [7] ………….................................... 8

Figure 2. 7 RP used in oral and maxillofacial surgery [9] ............................................ 9

Figure 2. 8 CAD model of a scaffold produced using FDM [13] ................................. 11

Figure 2. 9 Collagen Molecule Representations: A. Space-fill B. Wire C.

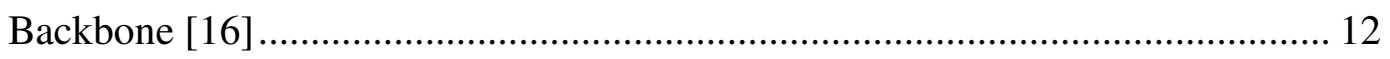

Figure 2. 10 Formation of an aldol cross-link between adjacent collagen

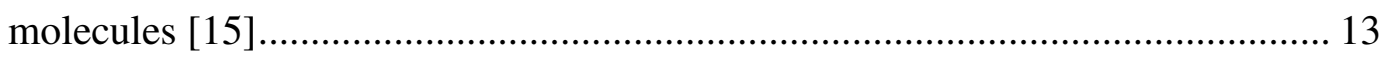

Figure 2. 11 Example of a gelatin monomer [20] .................................................... 14

Figure 2.12 Schematic of glutaraldehyde cross-linking protein chains [22] .................. 16

Figure 2. 13 SEM images of freeze-dried films frozen at: a. -20 degrees

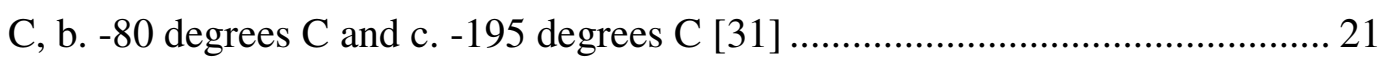

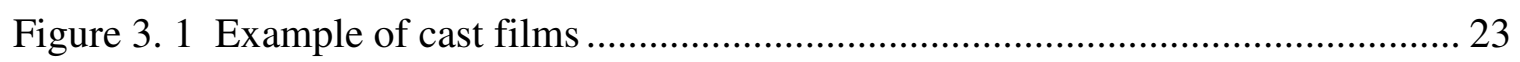

Figure 3. 2 Experimental set-up for the film dissolving test........................................ 26

Figure 3. 3 Frozen hydrated film immediately before freeze-drying process.................. 27

Figure 3. 4 Templates for selectively treating lines of varying widths.......................... 29

Figure 3. 5 Solidworks model used to build treatment templates................................... 30 
Figure 3. 6 Representation of a treated film in the first attempt at building

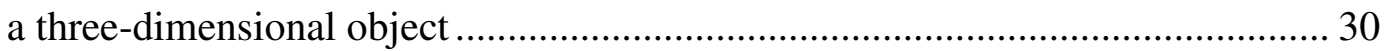

Figure 3. 7 Treatment using templates and ink before object assembly ...................... 31

Figure 3.8 Top and side views of films in containers ......................................... 31

Figure 3. 9 Representation of a treated film ..................................................... 32

Figure 3. 10 Freeze-dried films before stacking and hydrating ................................ 32

Figure 3.11 Stacked films before hydration, immediately after hydration, and 30 minutes after hydration. ............................................................... 33

Figure 3. 12 Unfreeze-dried films before and immediately after hydration ................... 33

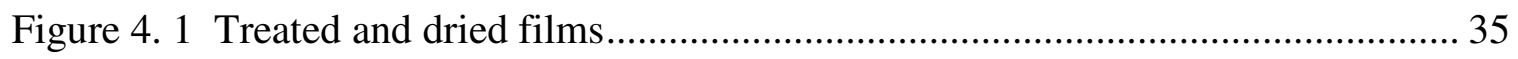

Figure 4. 2 Films produced by Treatment Protocol 2 ............................................. 36

Figure 4. 3 Films immediately after glutaraldehyde treatment ................................. 39

Figure 4. 4 Remaining treated lines of varying widths after dissolution of

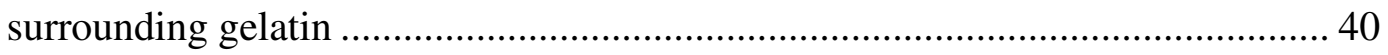

Figure 4. 5 Films immediately after adding water ............................................. 41

Figure 4. 6 Results of freeze-dried film assembly - top and bottom views................... 42

Figure 4. 7 Top view, magnified top view, and side view of the unfreezedried three-dimensional construct........................................................... 43 


\section{CHAPTER 1. INTRODUCTION}

\subsection{Need for the Study}

The applications for the technology developed in this study are vast. Modeling human tissue may increase physiological understanding and can even result in pharmaceutical production through functional cellular systems. Such pharmaceuticals may include essential proteins that allow the human body to function correctly. A number of methods of producing these proteins have been successfully investigated. One method employs genetic alteration to cause bovine or mice to produce these proteins through lactation [1]. However, a more efficient, sustainable, and animal-friendly method is preferred. Simulation of the mammary gland provides an avenue for the safe and efficient production of these proteins. This is just one of many possible applications of building working biological models.

Other applications of this technology may include simulating hormone glands, vascular networks, or other biological systems. A rapid fabrication method for building these structures is necessary to quickly advance research and development in this area. This study investigates the feasibility of such a process.

\subsection{Objective}

The objective of this study is to demonstrate and define a rapid prototyping process using glutaraldehyde treated films for the purpose of creating any three-dimensional structure for cell culture. It is demonstrated that it is feasible to produce a three-dimensional object by layering glutaraldehyde treated gelatin films. The applications and limitations of this process are 
explained. Finally, important questions and suggestions are identified that encourage further research. 


\section{CHAPTER 2. LITERATURE REVIEW}

The methods carried out in this study require basic knowledge of the following topics in this chapter. Rapid prototyping, collagen and gelatin materials, gelatin treatment methods, and freeze-drying are topics that allow for a richer understanding of the current study. A review of these topics has also been necessary to the development of this project.

\subsection{Rapid Prototyping}

Rapid prototyping (RP) is a method of creating a structure quickly based on a computer aided design (CAD) model. Alternative names for RP are: layer manufacturing, direct CAD manufacturing, and solid freeform fabrication (SFF) [2]. Many RP processes have been developed to quickly create parts, tools, or models of multiple shapes and materials. The RP processes that are relevant to this study are the material-addition RP technologies. These processes all work on the premise of dividing a computer designed three-dimensional object into multiple thin cross-sections, printing these cross-sectional shapes onto thin layers of material, and physically building the object with these layers.

\subsubsection{RP Methods}

The RP methods that will be discussed in this review are: stereolithography, solid ground curing, droplet deposition manufacturing, laminated object manufacturing, fused-deposition modeling, selective laser sintering, and three-dimensional printing.

Stereolithography, solid ground curing, and droplet deposition manufacturing processes utilize a liquid starting material. Stereolithography (SLA) was introduced by 3D Systems in 
1988 [3]. The process begins with a vat of photosensitive liquid polymer. A platform resides just underneath the surface of the liquid polymer, exposing the thin liquid layer to a laser mounted directly above. The scanning laser solidifies the coordinates that have been programmed into the system. Once the shape is solidified, the platform lowers to expose a new layer. A sweeper moves across the polymer to smooth the surface for the next laser treatment. As this process continues, a three-dimensional object is constructed.

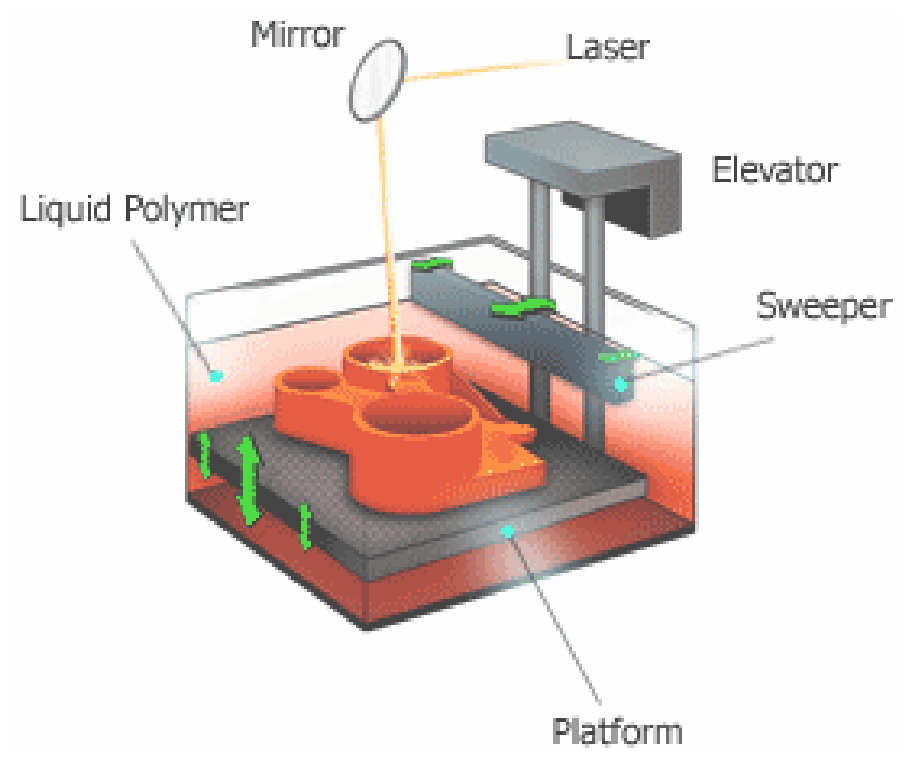

Figure 2. 1 Schematic diagram of the SLA process [2]

The solid ground curing (SGC) process is more complicated than that of SLA. The scanning laser is replaced with an ultraviolet light and a mask containing a negative image of the desired solidified shape. This mask is produced by electrostatically positioning toner in the desired cross-sectional image onto a glass plate before each curing cycle. The toner develops a charge pattern that is written with an electron gun [4]. The machine sprays a thin layer of photopolymer resin onto a platform. The resin that is unprotected by the mask is hardened by the ultraviolet lamp. Wax with a relatively low melting temperature is also used as a filler and 
support for subsequent liquid polymer layers. The surface of the wax and cured polymer are milled flat and vacuumed before the addition of the next polymer layer. This wax may be melted and removed from the part after the process is complete [3].

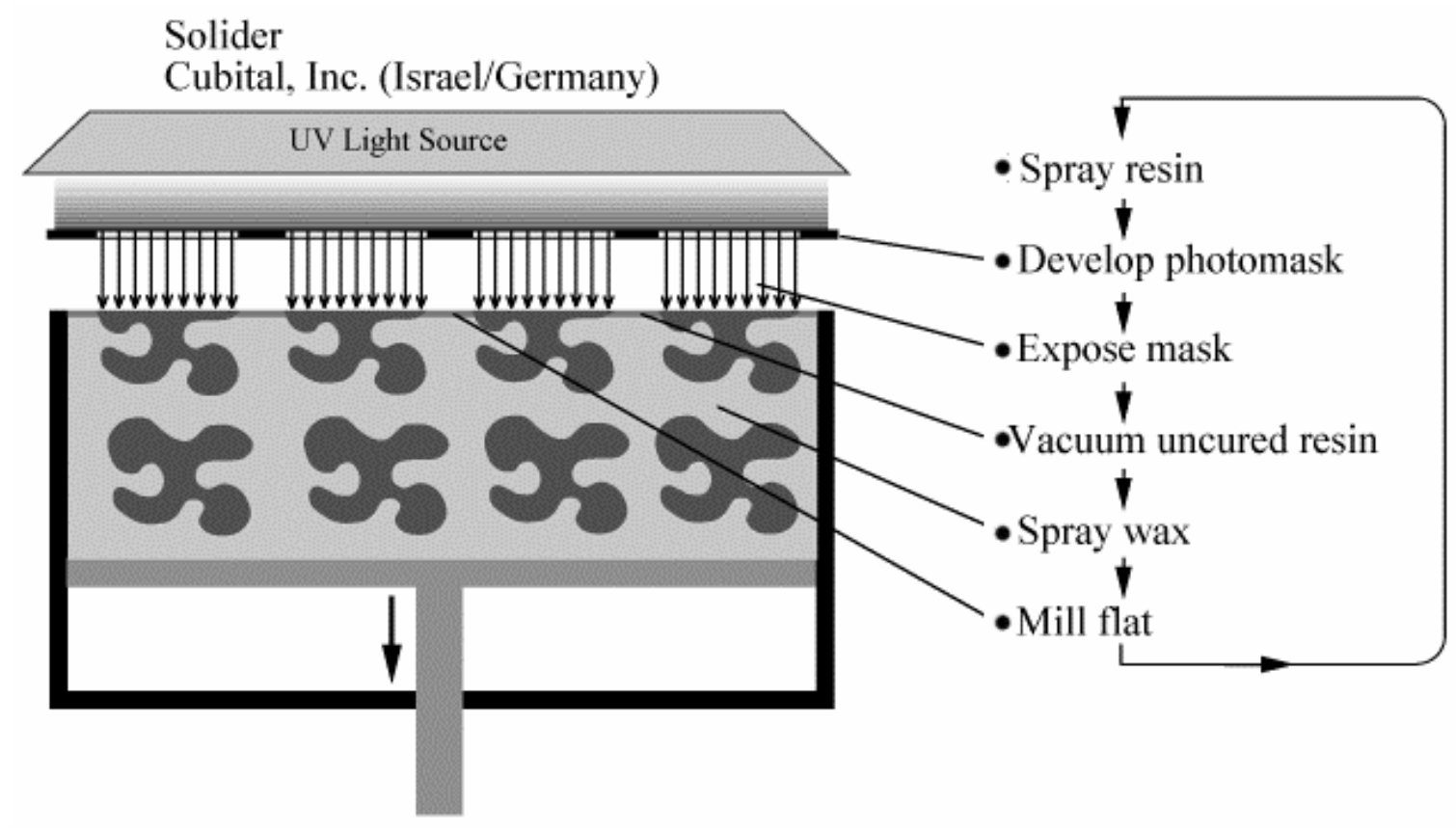

Figure 2. 2 Schematic of a Solid Ground Curing process [5]

Droplet deposition manufacturing (DDM) consists of dropping liquid material onto a flat surface. A spray nozzle drops the material according to coordinates programmed into the system. The droplet pattern forms the cross-sectional shape of the model layer. The material hardens on contact and the part is lowered for the next layer addition. Wax, thermoplastics, and metals with a low melting point can be used in this process. This method is also used in twodimensional applications such as printing solder onto circuit boards [3].

Laminated object manufacturing (LOM) and fused-deposition modeling (FDM) both use solid starting materials. LOM uses a laser to cut sheet stock according to the cross-sections of a CAD model. A heated roller is used to bind the underside of the stock to the underlying layer. 
The excess material is crosshatched for easy removal but remains in place to support the object until all of the layers are stacked. Materials used in this process are paper, plastic, cellulose, metals and fibersheet [3].

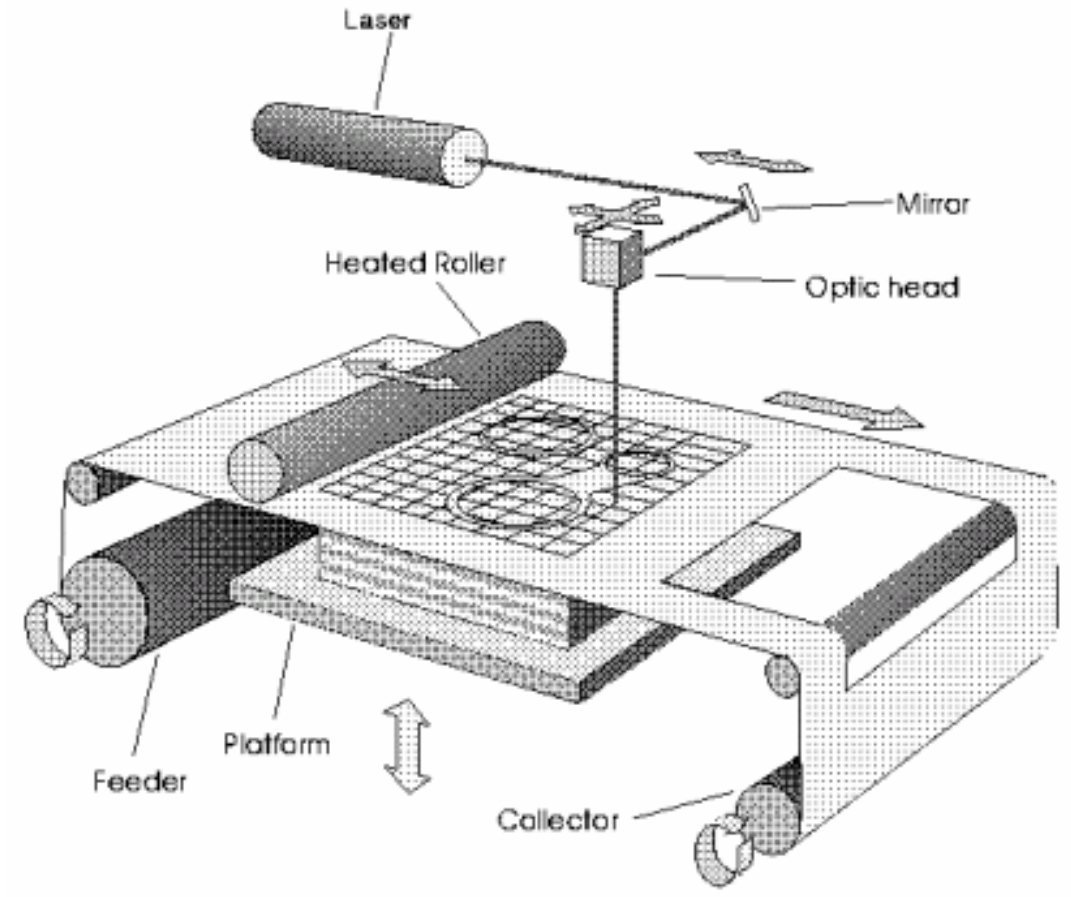

Figure 2. 3 Schematic of an LOM process [5]

Fused deposition modeling (FDM) starts with a solid filament of wax or polymer. An extrusion head heats the material to just above its melting temperature and extrudes it onto a platform. The material is quickly cold welded onto the cooler layer beneath. A second material may be dually extruded to create supports if needed. The materials used in this method are wax and several different polymers, including ABS, polyamide, polyethylene, and polypropylene [3]. 


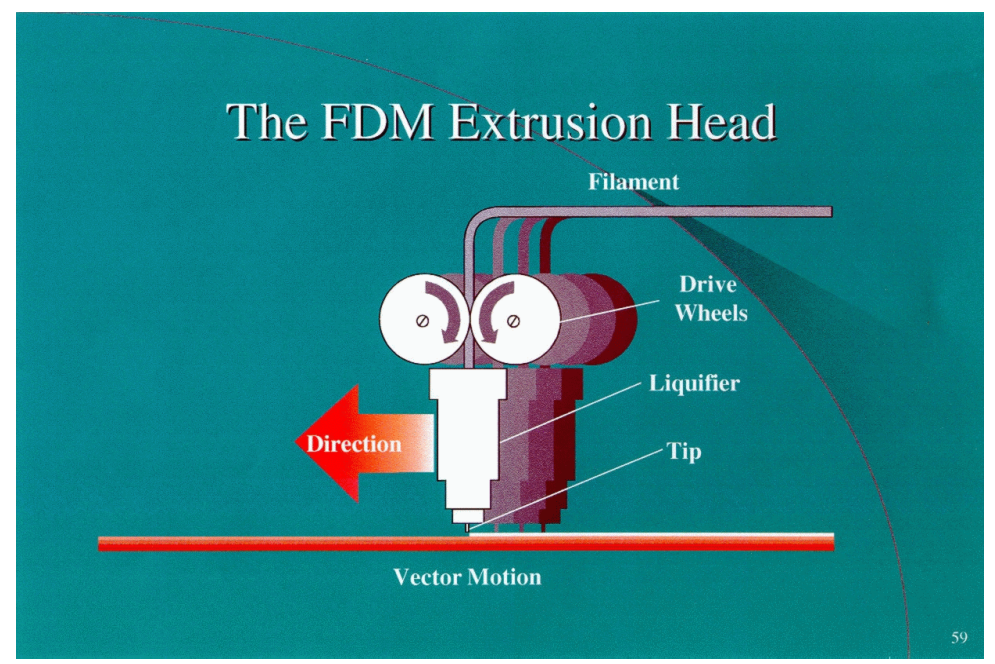

Figure 2. 4 Schematic of the FDM process [5]

Selective laser sintering (SLS) and three-dimensional printing (3DP) are both powderbased systems. SLS uses various powdered materials such as polyvinylchloride, polycarbonate, polyester, polyurethane, ABS, nylon, and investment casting wax [3]. The process begins with a thin layer of powder deposited onto a platform. The laser heats the pattern from the CAD model, binding the particles together. A new layer of powder is deposited, leveled, and laser-bound. This process continues until the part is complete. The excess powder helps support each layer and is removed when the part is finished.

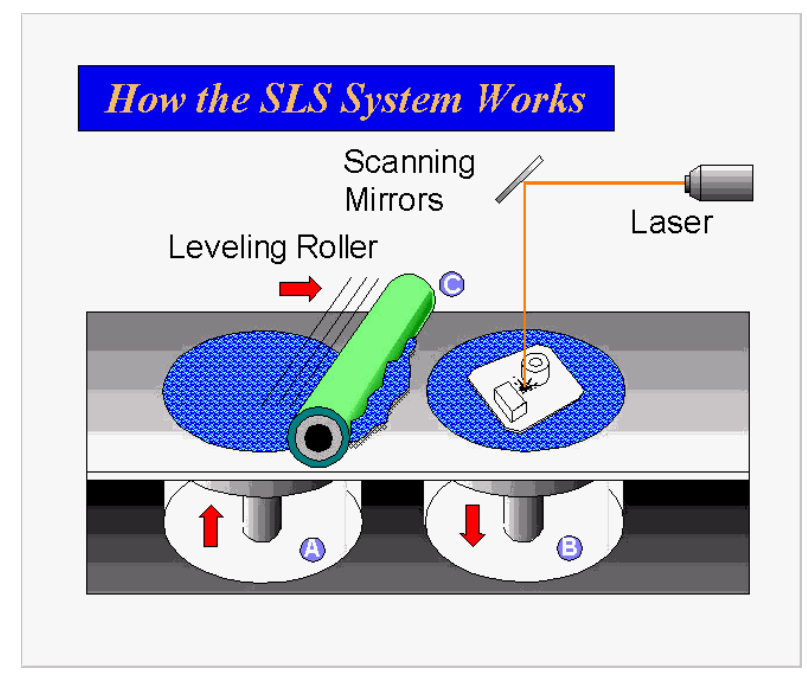

Figure 2. 5 Diagram of the SLS process [6] 
Three-dimensional printing also uses a base material in powder form. Instead of using heat to bind the particles, 3DP uses an adhesive bonding agent that is deposited from an inkjet head. The powdered materials that are used in this process include ceramics, metals, or cement. The bonding agents may include polymers, colloidal silica, or silicone carbide [3]. This RP method was the first to fabricate drug delivery devices.

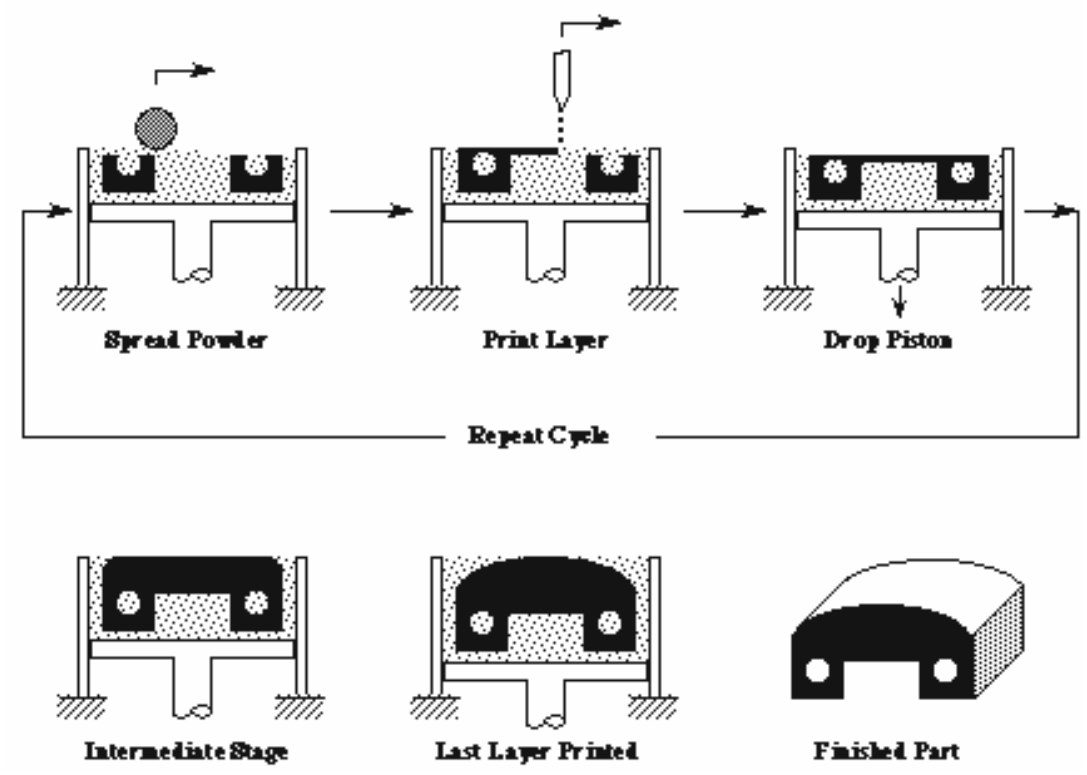

Figure 2. 6 3-Dimensional Printing Schematic [7]

\subsubsection{RP in Medical Applications}

Rapid prototyping has been effective in decreasing lead times and increasing the quality of manufactured parts. It has also become useful in the medical industry. Because RP uses digital information that describes a solid object, data from computerized tomography (CT) scans and magnetic resonance imaging (MRI) can be used to construct models of structures inside the body. These models have been used in a variety of applications including medical device design [4], simulated complex surgeries, analysis and planning of implant design, bone structure 
regeneration, instruction and communication aids for health professionals, and even forensic analysis $[8,9]$.

Figure 2.7 shows an example of RP used in surgical planning of facial structure replacement. This type of surgery requires careful planning because of the complex bone structure of the skull and the emphasis on appearance of the face. An SLA model of the patient's skull was used to prepare for implanting a titanium mesh to replace the damaged cheekbone. The left side of the patient's face was mirrored and provided a template for the titanium mesh design [9].
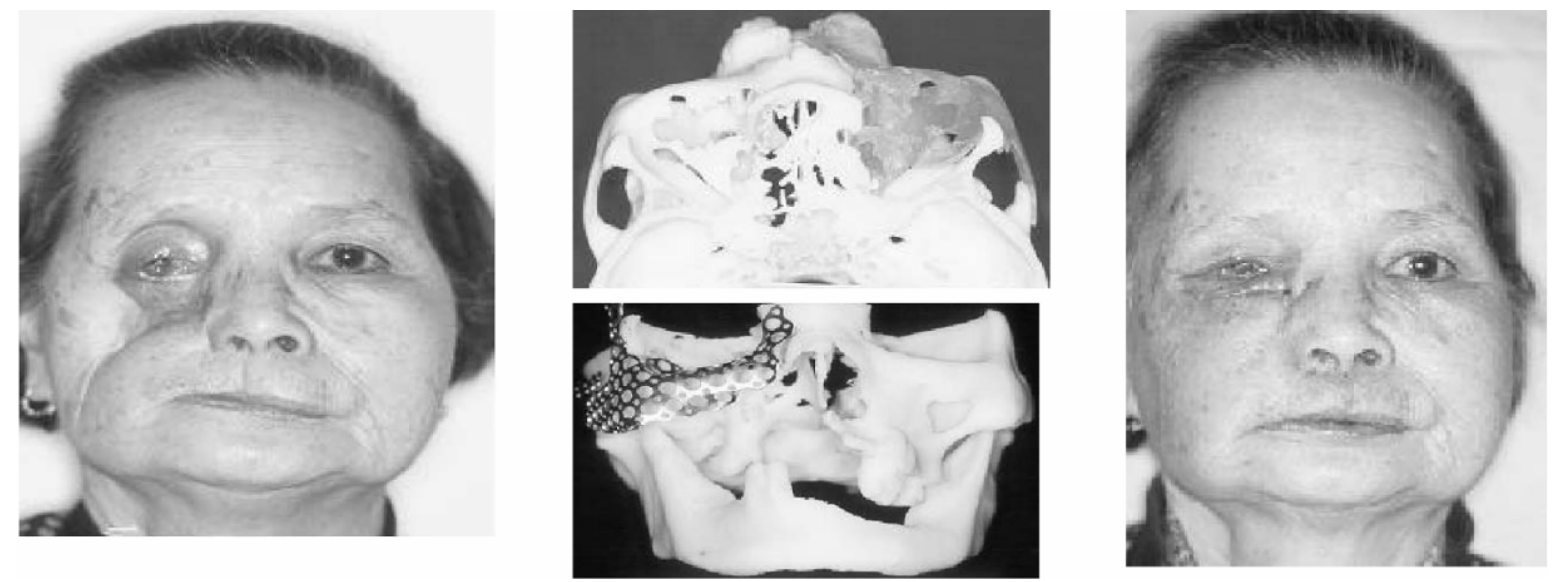

Figure 2. 7 RP used in oral and maxillofacial surgery [9]

\subsubsection{RP in Tissue Engineering}

Rapid prototyping has recently been employed as a scaffold fabrication method. A large area of tissue research focuses on development of implantable replacement tissue. A scaffold designed for this application serves as a temporary extracellular matrix (ECM) to guide the spread of seeded cells [10]. When cells have proliferated throughout the scaffold, the supporting material degrades and leaves only the desired tissue. 
Some scaffold fabrication methods include fiber bonding, solvent casting and particulate leaching, membrane lamination, melt molding, gas foaming, and freeze drying [10]. Disadvantages of these methods include long fabrication periods, poor repeatability, and irregular pore structure [9]. The layering method of RP allows for greater uniformity and control over the internal structure of scaffolds. RP can refine pore interconnectivity, size, and shape to increase cell proliferation, differentiation, and mobility.

A study was published by Lam et. al. in 2002 that described experiments in developing porous scaffolds using 3D printing. This process was chosen for the purpose of accurately producing repeatable interconnectivity and structure. A three powder blend made up of cornstarch, dextran, and gelatin was used as the based material. Distilled water was used as a binding agent. The structures contained pores of cylindrical and rectangular shapes of varying sizes. Post processing techniques of heating and soaking in water were also used [11].

Table 2. 1 Scaffolds produced using a 3DP method [11]

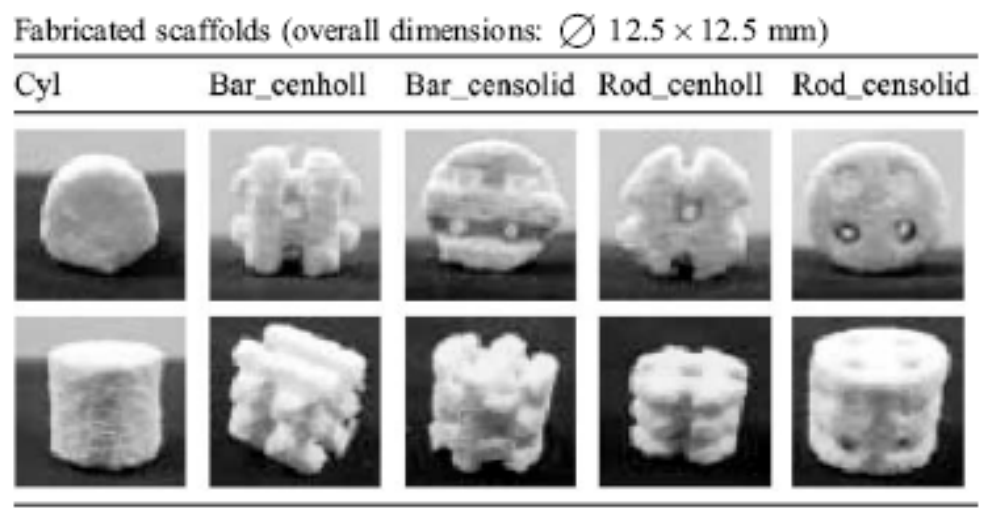

In 2005, Darling et. al. reported using an FDM process to construct scaffolds onto which chondrocytes had been successfully cultured. PCL was heated and deposited in $10 \mathrm{mil}$ or 7 mil 
diameter strands. This allowed for a scaffold with $99 \%$ connectivity and optimal porosity and pore size to be constructed [12].

In 2006, Xiaohong et. al. used RP to produce structures composed of hepatocytes and gelatin hydrogel. The process used was also similar to FDM. The starting material was made up of living cells and gelatin solution. Before deposition, the mixture was cooled to just below 20 degrees $\mathrm{C}$ for the purpose of rapid solidification and stability of the gel. The scaffolds were then soaked in glutaraldehyde and ultimately lasted for up to two months with living and functional cells [13].

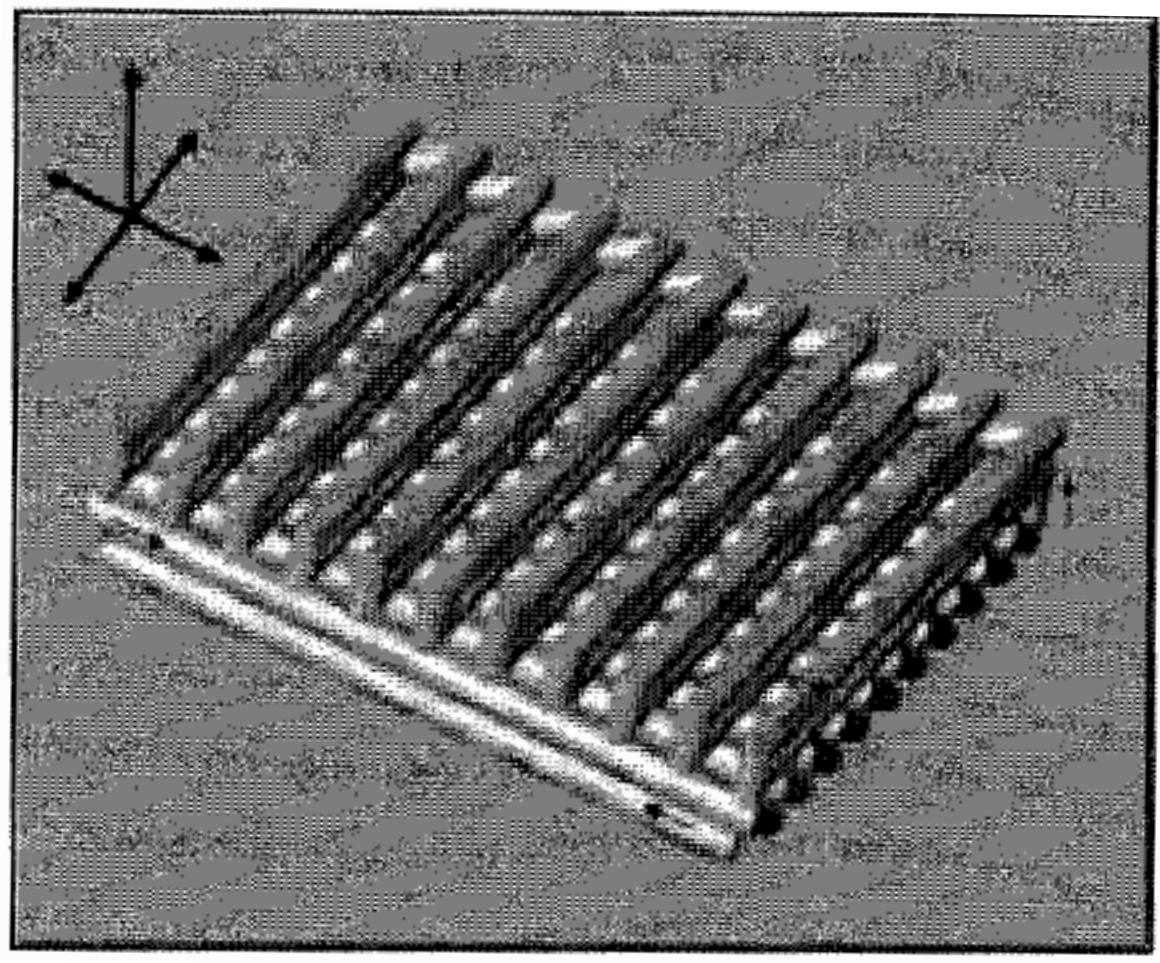

Figure 2. 8 CAD model of a scaffold produced using FDM [13] 


\subsection{Biomaterials}

Gelatin was used as the base material in the current rapid prototyping process. A review of the process for obtaining gelatin is appropriate for understanding gelatin properties. This process begins with collagen, the material from which gelatin is derived.

\subsubsection{Collagen}

Collagen is the most abundant protein found in the human body $[13,14]$. There are at least sixteen types of collagen that have similar structures. Different types of collagen contain segments in their molecular structure that cause the collagen molecules to form unique threedimensional structures. The skin consists of both Type I and Type III collagen [15].

\section{Collagen Molecule Representations}

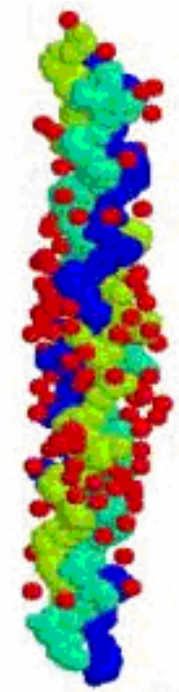

A

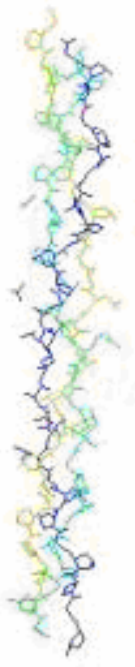

B

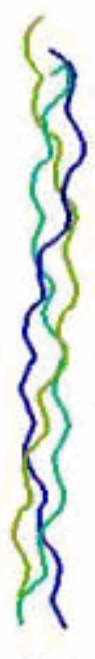

$\mathrm{C}$

Figure 2. 9 Collagen Molecule Representations: A. Space-fill B. Wire C. Backbone [16] 
The structure of a collagen molecule consists of three polypeptide chains formed into a triple helix. This structure is stabilized by interchain hydrogen bonds and covalent cross-links [14]. Each chain has a -Gly-Pro-X- repeating sequence where $\mathrm{X}$ can be any amino acid. The glycine residues form hydrogen bonds with the $\mathrm{X}$ residue of the neighboring chain. Collagen molecule bond by forming aldol cross-links between two lysine or hydroxylysine residues [15]. Figure 2.10 shows a diagram of this linkage formation. In fibrillar collagen, the molecules are organized in a striated structure that provides strength and stability to surrounding tissues and supports movement and flexibility.

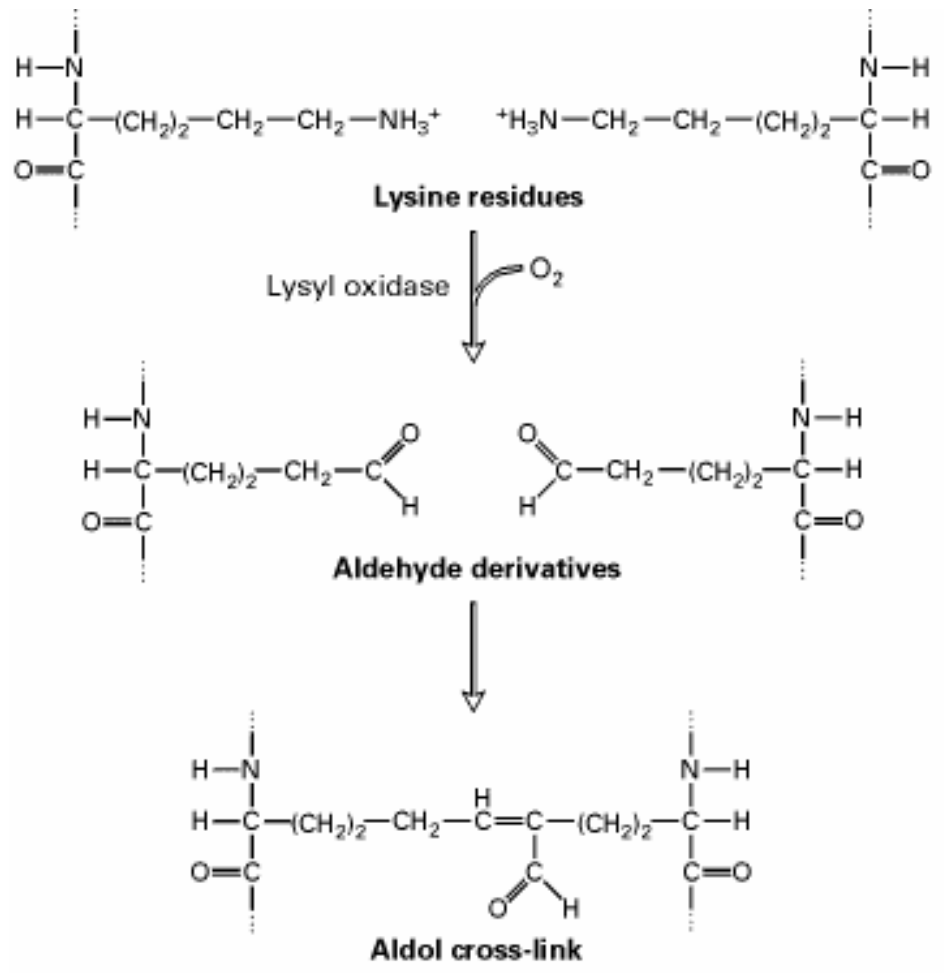

Figure 2. 10 Formation of an aldol cross-link between adjacent collagen molecules [15]

Collagen can be broken down to produce gelatin by denaturation. This process consists of exposing collagen to high temperatures, solutions of extreme $\mathrm{pH}$ values, or specific enzymes 
$[18,19]$. The main chain peptide bonds and the covalent cross-links are ruptured during denaturation, breaking down the structure of collagen. The gelatin extracted from the resulting material has almost the same amino acid configuration.

\subsubsection{Gelatin}

Gelatin is mixture of single or multi-stranded polypeptides. Each strand contains anywhere from 300 to 4000 amino acids, the most common being glysine, proline, and hydroxyproline [20]. Figure 2.11 shows an example a gelatin monomer within a polymer chain. Two types of gelatin exist that have different isoelectric points and denaturation processes. Type A or acidic gelatin is derived from acid-cured collagen. Type B or basic gelatin if derived from lime-cured tissues. Type A gelatin has an isoelectric point of nine, exhibiting a net negative charge in solutions with a $\mathrm{pH}$ above nine and a net positive charge in solutions with a $\mathrm{pH}$ below nine. Type B gelatin has an isoelectric point of five [21].

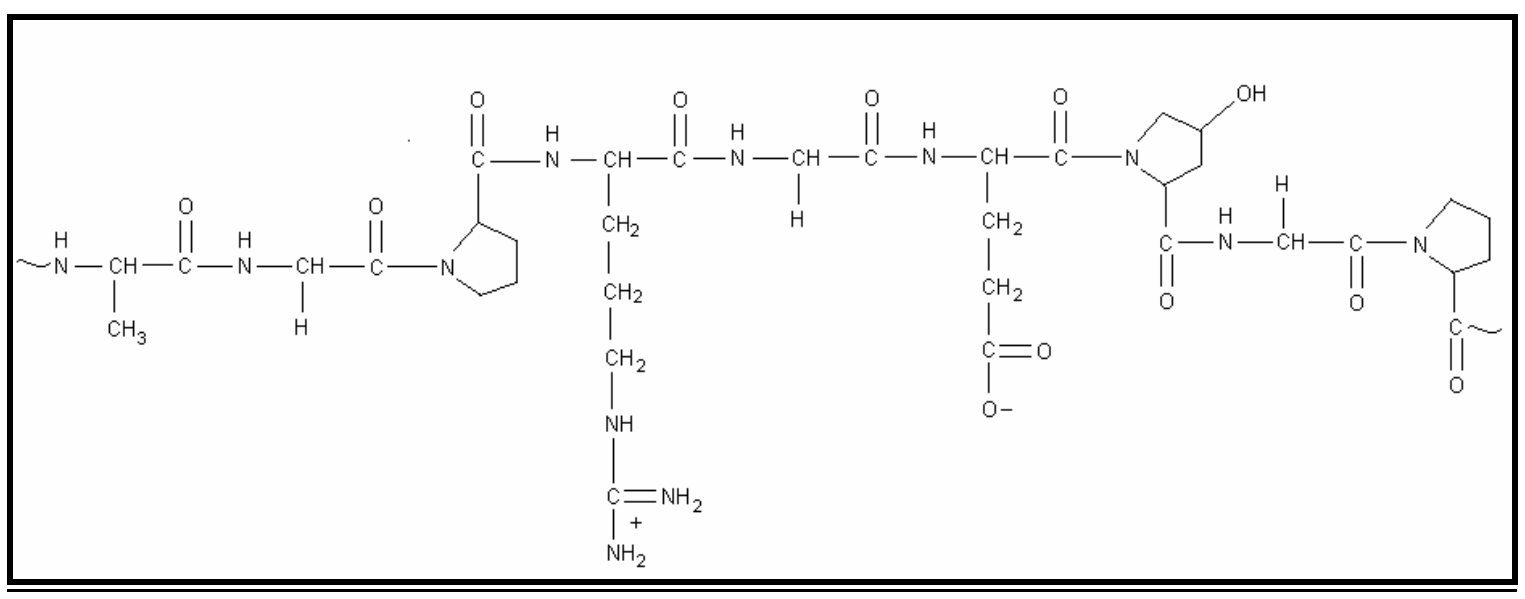

Figure 2. 11 Example of a gelatin monomer [20]

Gelatin is water soluble, rapidly biodegradable, and has high molecular weight [22].

When hydrated, gelatin acts as a sponge and swells to accommodate water molecules within its 
structure. Preparation of gelatin consists of dissolving gelatin particles into warm water. The solution becomes viscous and hardens as it cools. When the gelatin solution undergoes a sol-gel transition, thermoreversible networks form and are stabilized by hydrogen bonds. These bonds form at the junction zones of associated gelatin strand helices [23]. The stability of gelatin increases with increased association of helices. The strength of gelatin is defined by its Bloom number, the amount of mass in grams needed to push a $12.5 \mathrm{~mm}$ diameter plunger $4 \mathrm{~mm}$ into gelatin [21].

Gelatin has been useful in biomedical and non-biomedical applications, including food technology and photography. The most common biomedical applications utilizing gelatin are bioadhesion and sustained drug release. It has been used as a stabilizer, thickener, texturizer, and as a coating layer [19]. It has also, more recently, been used in artificial skin and neuron regeneration applications [24].

\subsection{Gelatin Modification Methods}

Gelatin's low melting temperature and high degradation rate limits its applications in human physiological simulation and cell culture methods. However, gelatin is a biocompatible substance that is inexpensive to obtain and easy to manipulate. Therefore, a number of methods for increasing the strength and stability characteristics of gelatin have been developed. The methods discussed here are cross-linking, composite development, and mechanical methods.

\subsubsection{Cross-linking}

Cross-linking is a material modification method that covalently binds molecules. This treatment method can increase the strength and stability of otherwise rapidly degrading gelatin. 
Two types of cross-linkers exist: homobifunctional and heterobifunctional cross-linkers.

Because homobifunctional cross-linkers contain two identical reactive groups, they are used in one-step reaction procedures that stabilize the material quaternary structure. Heterobifunctional cross-linkers require a two-step procedure. These cross-linkers possess two different reactive groups that bind different types of proteins. Heterobifunctional cross-linking procedures require at least two steps to form the appropriate bonds [25]. The cross-linking agents discussed here are glutaraldehyde, formaldehyde, carbodiimide, and genipin.

\subsubsection{Glutaraldehyde}

Glutaraldehyde, a bifunctional cross-linker, is the most commonly used cross-linking agent. This aldehyde is also used as a sterilant. In tissue scaffold and bioreactor development, this provides the advantage of eliminating the necessity of sterilization before cell culture [18]. However, glutaraldehyde is also toxic in large concentrations, which poses disadvantages of special waste removal methods and cytotoxicity considerations.

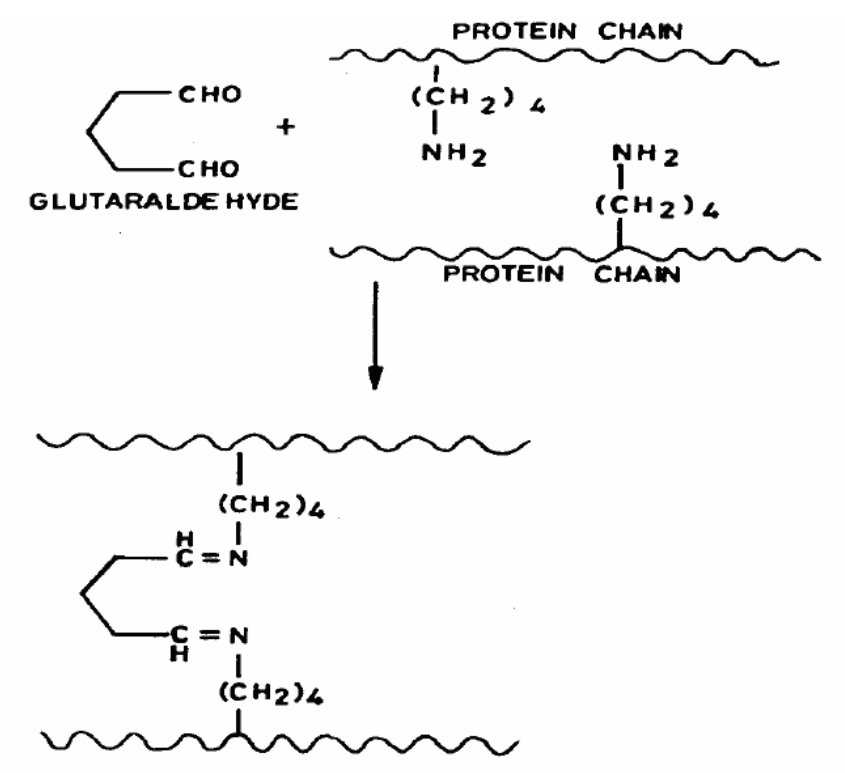

Figure 2.12 Schematic of glutaraldehyde cross-linking protein chains [22] 
Treatment methods and material characteristics of glutaraldehyde cross-linked gelatin have been studied by Lou et. al. [19]. The results concluded that higher concentrations of glutaraldehyde can decrease toughness and elasticity while increasing stiffness. Also, higher concentrations of gelatin increased the ultimate strength of the product, while insignificantly affecting the stiffness and elasticity. Cross-linking also increases the denaturation temperature of gelatin and decreases the denaturation enthalpy. These altered material properties provide gelatin with the ability to withstand environments simulating in vivo conditions [14].

A few methods, observations, and explanations have been noted when treating gelatin with glutaraldehyde. First, an explanation of the light color on a treated gelatin film is offered. This yellow tint is due to the aldamine linkage (also referred to as a Schiff linkage) between the free amino groups of protein and glutaraldehyde. Second, it was determined that the majority of the linkages involve the gelatin's residual protein groups, as opposed to the other groups. Finally, when treating with glutaraldehyde, glycine is often used to remove residual free $-\mathrm{CHO}$ groups that are not involved in the cross-linking process. If these groups are not blocked or removed, the resulting cross-linked material may exhibit a chemo-attractant property [19].

\subsubsection{Formaldehyde}

Formaldehyde is a cross-linker similar to glutaraldehyde. It is the simplest aldehyde that forms cross-links by creating methelene bridges between reactive proteins. The functional groups that are reactive in formaldehyde are amido, guanidine, thiol, phenol, imidazole and indolyl groups. Compared to glutaraldehyde, formaldehyde cross-links proteins at a slower rate but penetrates the cross-linking material ten times faster, providing an advantage for three- 
dimensional cross-linked structures [25]. However, formaldehyde also exhibits cytotoxic affects in cell cultures when used as a gelatin cross-linker.

\subsubsection{Carbodiimide}

Carbodiimides are heterobifunctional cross-linkers. They are also zero-length crosslinkers that promote direct coupling, but are not part of the linkage. Lee et. al. compared carbodiimide EDC cross-linking to glutaraldehyde cross-linking and found that the resulting materials were similar except for the increased extensibility and elasticity of EDC.

Carbodiimides have also been found to create both short range intermolecular and intramolecular cross-links, but create no long range intermolecular links [27].

\subsubsection{Genipin}

Genipin cross-linking is comparatively slower than carbodiimide cross-linking. Also, genipin has been found to create long range intermolecular cross-links as well as short range intermolecular and intramolecular links [28]. Genipin cross-linked gelatin also exhibited lower toxicity than carbodiimide cross-linked gelatin. This could be foreseen by considering that genipin is a natural substance extracted from gardenia fruits [18].

\subsubsection{Gelatin Composites}

A number of materials have been utilized for making composite reinforced gelatin. Some of these include montmorillonite, hydroxyapatite, tricalcium phosphate, and carbon fiber [23]. The two composites discussed here are fibrin-chitosan-gelatin composites and hydrotalcitegelatin composites.

Composite structures also allow gelatin to maintain its biocompatible properties while adopting a more stable structure. One composite studied recently was a fibrin-chitosan-gelatin 
mixture treated with glutaraldehyde. The fibrin web was infiltrated with gelatin and bound by chitosan, a non-toxic, biodegradable polymer. Chitosan has a high charge density in solution, which allows it to remove toxic and contaminating materials from negatively charged living tissues. This combination produced a material with desirable characteristics for wound dressing applications including: increased porosity, stability, and water absorption capacity [24].

The addition of hydrotalcite to hydrogels has also been studied with special attention give to swelling properties of treated films. Lee et. al. found that adding a small amount of intercalated hydrotalcite to gelatin could decrease the swelling ratio of hydrated gelatin to dry gelatin. However, above this amount, a positive correlation exists between hydrotalcite concentration and the swelling ratio [23].

\subsubsection{Physical Treatment}

Gelatin properties may also change due to mechanical or radiation stimulation. Bigi et al. investigated a drawing process in which gelatin films were elongated over time. The process promoted the chain alignment and resulted in improved mechanical properties for films stretched up to two times their original length [14]. Cross-linking has also been induced using radiation methods [30].

\subsection{Freeze-drying}

Freeze-drying, also known as lypholization, has been widely utilized in materials and tissue engineering, as well as everyday applications such as food preservation. It is a preservation technique that allows for hydrated material to retain its structure after removal of water. 


\subsubsection{Process}

The freeze-drying process begins with the freezing of a hydrated material. The material is then placed in a chamber in which the temperature and the pressure can be altered. The temperature is reduced and maintained below the melting temperature of water and a reduction in pressure follows. At this low temperature and pressure, the ice in the chamber sublimates and leaves the host material dry while remaining in its swelled structure.

\subsubsection{Considerations}

This process offers many advantages in bioengineering. Freeze-drying can increase stability and the rate of solubility of gelatin. In the present application, these properties will prove useful to creating a more robust three-dimensional object in less time.

In scaffold development applications, the water has been used as a porogen that aids in the development of pores, interconnectivity, and uniformity in scaffold materials. It has been shown that the morphology of these pores can depend on the freezing temperature of the hydrated gelatin. The explanation for this correlation is that the colder temperatures do not give water enough time to crystallize. The water freezes at temperatures below -50 degrees $\mathrm{C}$ while forming ice crystal nuclei, creating small pores and a thinner structure. A sample frozen at -20 degrees $\mathrm{C}$ has exhibited a three-dimensional morphology with round pores and a puffy or spongy morphology. It is hypothesized that the heat transfer dynamics of the higher freezing temperature allows the water to fully crystallize and support the material thickness [31]. The following figures show freeze-dried films processed at varying freezing temperatures. 


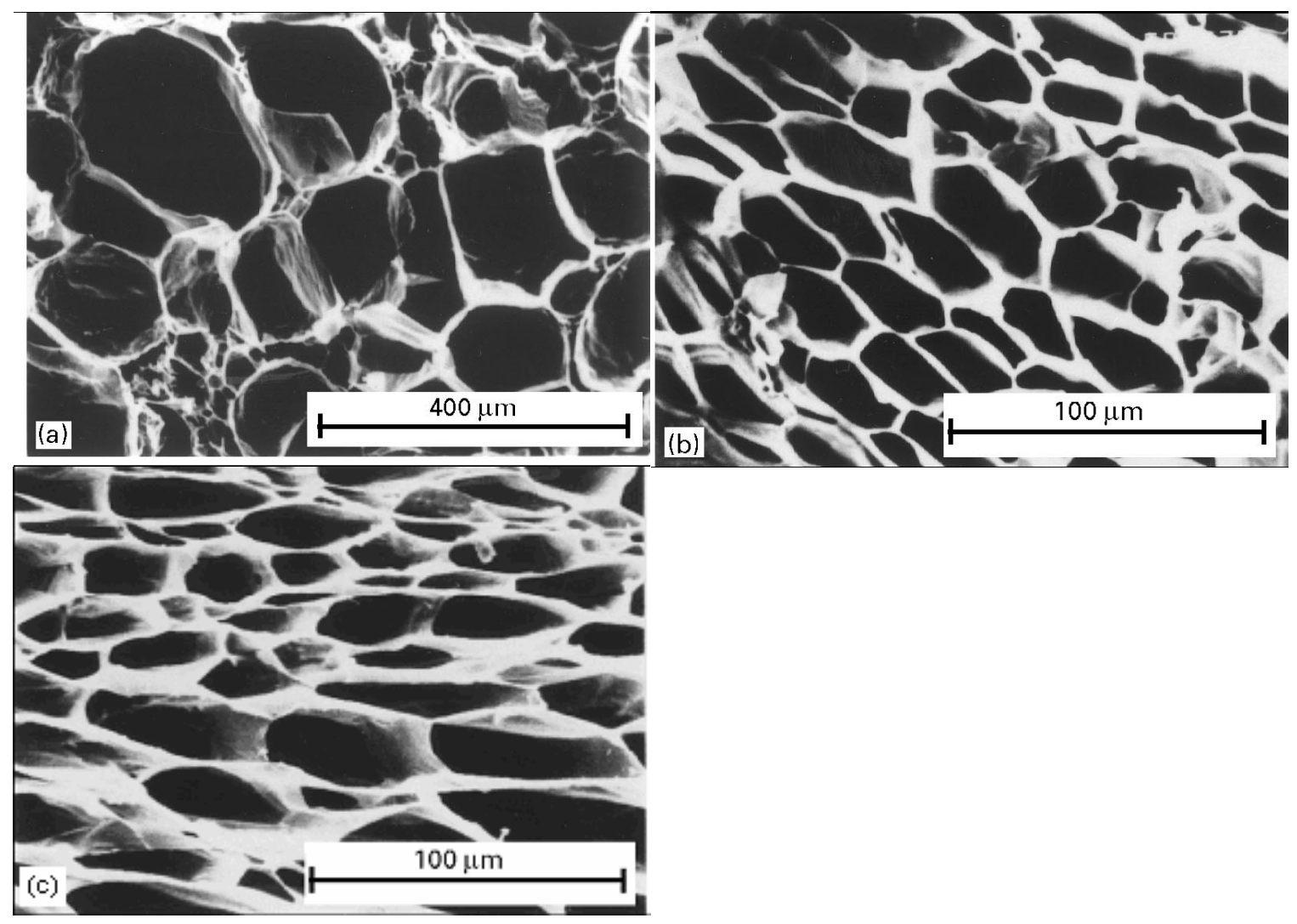

Figure 2. 13 SEM images of freeze-dried films frozen at: a. -20 degrees C, b. -80 degrees $C$ and c. -195 degrees $C$ [31] 


\section{CHAPTER 3. EXPERIMENTAL PROCEDURES}

Five experiments were done in for the purpose of determining possibilities, limitations, and effective practices using a printing method for treating films and constructing a threedimensional object. Observations were made regarding the strength and stability of films with varying process conditions.

\subsection{Outline Selective Treatment Experiment}

The purpose of this experiment was to observe how gelatin films behave when selectively treated with different shapes and varying concentrations of glutaraldehyde. This experiment aided in determining the processing conditions of the films including the rinsing temperature, rinsing time, glutaraldehyde concentration, gelatin concentration, and glutaraldehyde application method.

\subsubsection{Materials and Equipment}

Materials used included: Porcine skin 300 Bloom Type A Gelatin (Sigma Chemical Co., St. Louis, MO), Glutaraldehyde $2.5 \%$ concentration (Sigma), Glycine powder (Sigma), Masking tape (Staples), Polycarbonate plastic block, Micro-Balance (Acculab, Edgewood, NY), Desiccator.

\subsubsection{Procedure}

\subsubsection{Casting films}

Teflon blocks were used as the base material for casting the gelatin films. Four squares $(30 \times 30 \times .1 \mathrm{~mm})$ were formed with a double layer of masking tape. Milli-Q water was heated 
to 65 degrees $\mathrm{C}$ and the appropriate amount of Type A gelatin powder was added to produce a solution with a gelatin concentration of $10 \%$ by weight. To cast one block, 10 grams of gelatin powder was added to $100 \mathrm{ml}$ of water. The films were cast by using a micropipette distribute 1 $\mathrm{ml}$ of liquid evenly within the each square. The total volume of each cast square was $1 \mathrm{ml}$. The procedure was repeated using a gelatin concentration of $20 \%$ by weight.

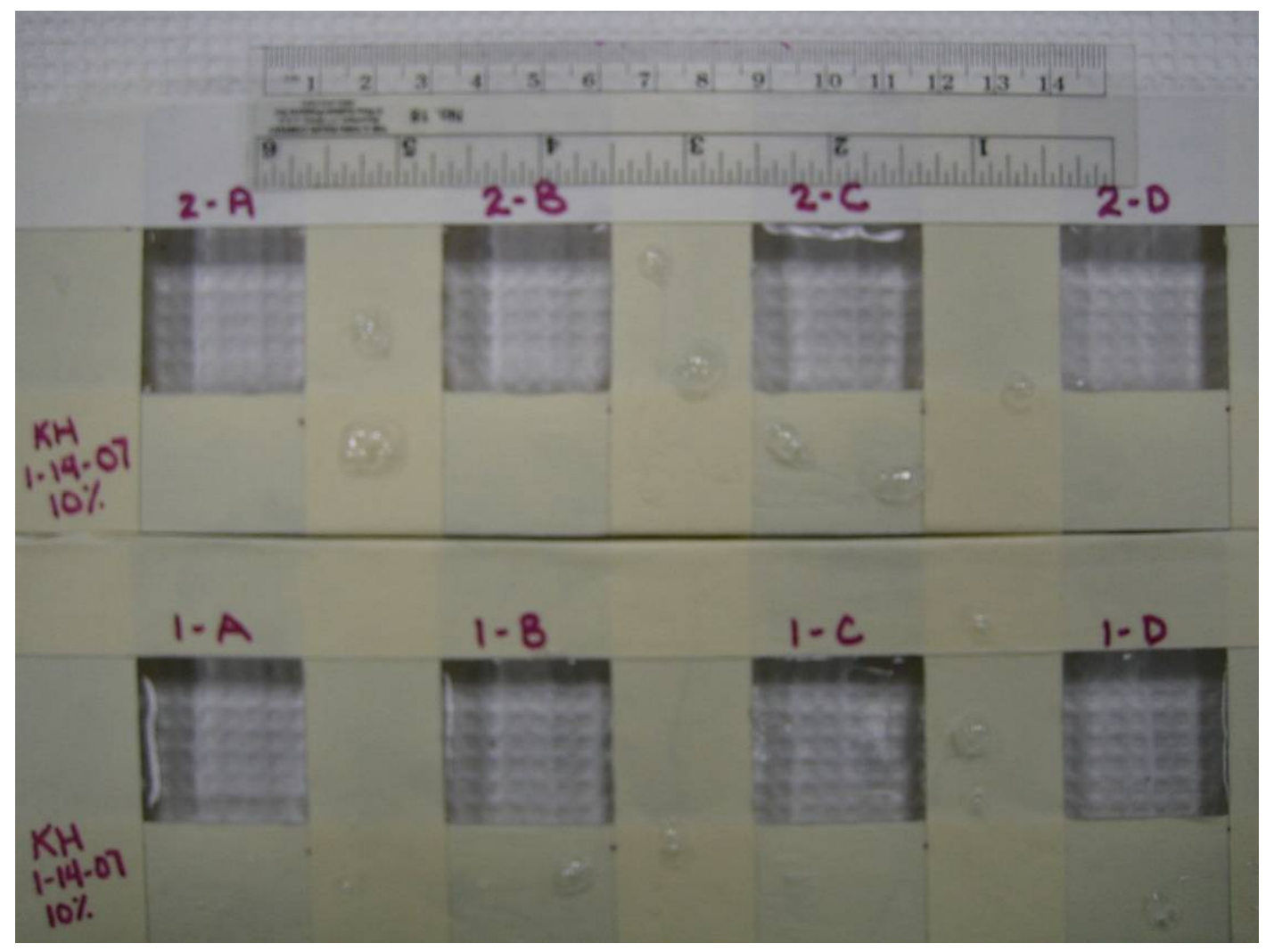

Figure 3. 1 Example of cast films

\subsubsection{Treatment Protocol 1}

Once the films were cast and dried overnight, they were removed from the casting blocks, trimmed of residual tape, and each placed a Petri dish. If treatment was not administered immediately, the films were placed in the desiccator to prevent rehydration and reformation. $2.5 \%$ concentration glutaraldehyde was diluted to $0.3 \%$ concentration under the hood. The GTA 
treatment was administered by using a $1 \mathrm{ml}$ micropipette as a free-hand "drawing tool". Shapes drawn on the films included triangles, squares, circles, and a square mesh. The films were treated for 20 minutes (beginning at the completion of the last shape) and agitated in milli-Q water at room temperature for three hours, aspirating off and replacing the water every hour. A solution of $1 \%$ glycine concentration by weight was prepared by weighing 5 grams of glycine powder into $500 \mathrm{ml}$ of milli-Q water. The films were then treated with glycine for 2 hours for the purpose of removing free carboxyl groups. The glycine was aspirated off after which the rinsing/agitation procedure was repeated for 2 hours. The films were left to dry overnight after the final rinse. They were removed from the Petri dishes when dry and visually and tactilely observed.

\subsubsection{Treatment Protocol 2}

Treatment Protocol 2 was developed in response to the unfavorable results of Treatment Protocol 1. This revised protocol lowered the duration of rinsing and the temperature of the milli-Q water and glycine. It also eliminated the mechanical stimulus of agitation. Only $10 \%$ gelatin concentration films were cast in this procedure. The casting protocol was not altered. The water and glycine were kept at 0 degrees Celsius by retrieving liquid from partially frozen solutions. The first rinse lasted only 1 hour with water replacement every 20 minutes. The glycine treatment lasted for only one hour without a solution change. The final rinse also lasted one hour with water replacement every 20 minutes. The films were left to dry overnight after the final rinse and aspiration. They were removed from the Petri dishes when dry and visually and tactilely observed. 


\subsection{Film Dissolution Experiment}

The purpose of this experiment was to observe how well the treated portions of the films remained intact and true to their original shape when the untreated film was dissolved. The experiment demonstrated that selectively treated gelatin film will remain intact after the dissolution of untreated film and provided an estimate of the time required for such a process. The test also compared the dissolution characteristics of films prepared with Treatment Protocol 1 and Treatment Protocol 2.

\subsubsection{Materials and Equipment}

Three labeled beakers and a hot plate were utilized in this experiment. Milli-Q water was used as the solvent. Two films prepared with Treatment Protocol 1 (one of 10\% gelatin concentration and one of $20 \%$ gelatin concentration) and one film of $10 \%$ concentration prepared with Treatment Protocol 2 were tested.

\subsubsection{Procedure}

Each beaker was filled with $1 \mathrm{~L}$ of milli-Q water and heated to 37 degrees C. One film was submerged into each beaker. The films were monitored every 10 minutes for one hour; then every 30 minutes until the untreated film had completely dissolved. 


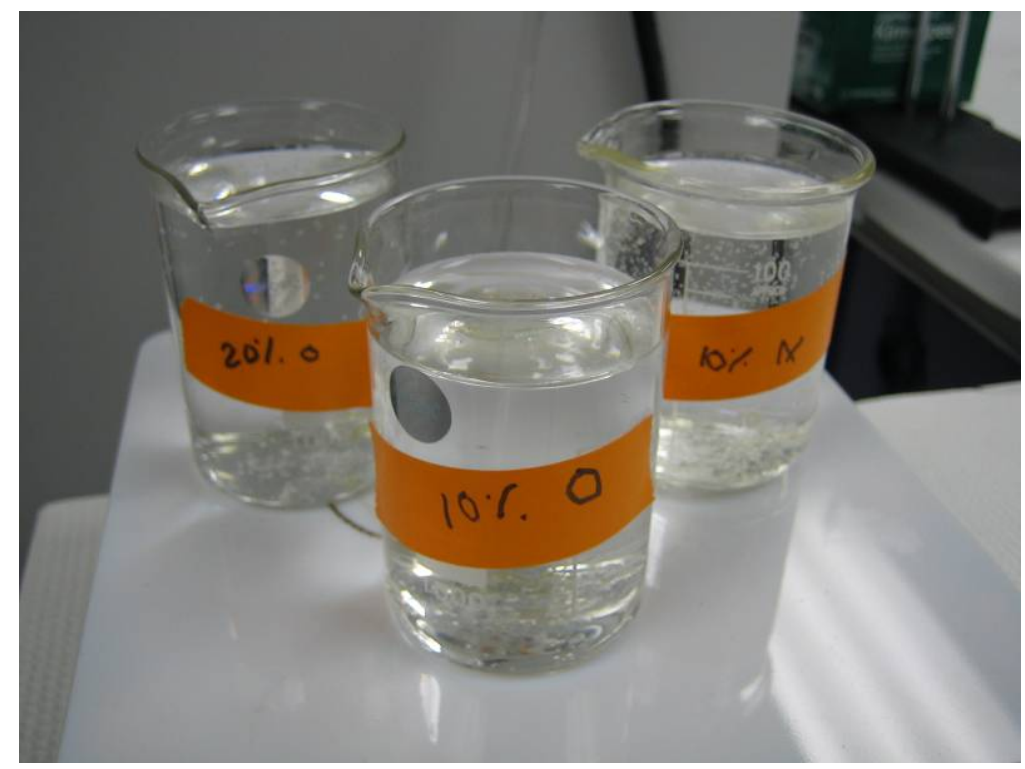

Figure 3. 2 Experimental set-up for the film dissolving test

\subsection{Treating Freeze-dried and Unfreeze-dried Films}

The purpose of this experiment was to determine the behavior of freeze-dried films when selectively treated and possible benefits of freeze-drying in the resulting films. Observations of the ease of treatment as well as effectiveness of treatment were noted.

\subsubsection{Materials and Equipment}

All materials and equipment from Experiment 3.1 were used. A freeze-dryer purchased from Millrock and dry ice (frozen carbon dioxide) purchased from Albertsons were also used. Paint brushes from Aaron Brothers were used for applying glutaraldehyde. A triangular pyramid was first modeled with Solidworks three-dimensional modeling software and sliced at .1 mm sections. The sections were made into a drawing template that allowed the shape to be anchored inside a "treatment window". This template was used as a guide for treating films. Examples of treatment templates are in Appendix A and Section 3.5. 


\subsubsection{Procedure}

This experiment used Treatment Protocol 2 with a few exceptions. The films were cast as they were in previous experiments. They were then saturated, frozen in dry ice (carbon dioxide with a surface temperature of -78.5 degrees $\mathrm{C}$ ), and freeze-dried in a freeze-dryer obtained from Millrock. The resulting films had a thickness of about 1.5 times the thickness of the unfreeze-dried films. This increase in thickness gave them a texture and nontransparent characteristic similar to parchment paper. The films were treated along side films that did not go through the freeze-drying process. Thin paint brushes were used to deposit glutaraldehyde onto the films by dipping paint brushes into the GTA solution and dabbing it onto the film surface. This was done three times during the 20 minute GTA treatment interval for the purpose of replacing absorbed glutaraldehyde.

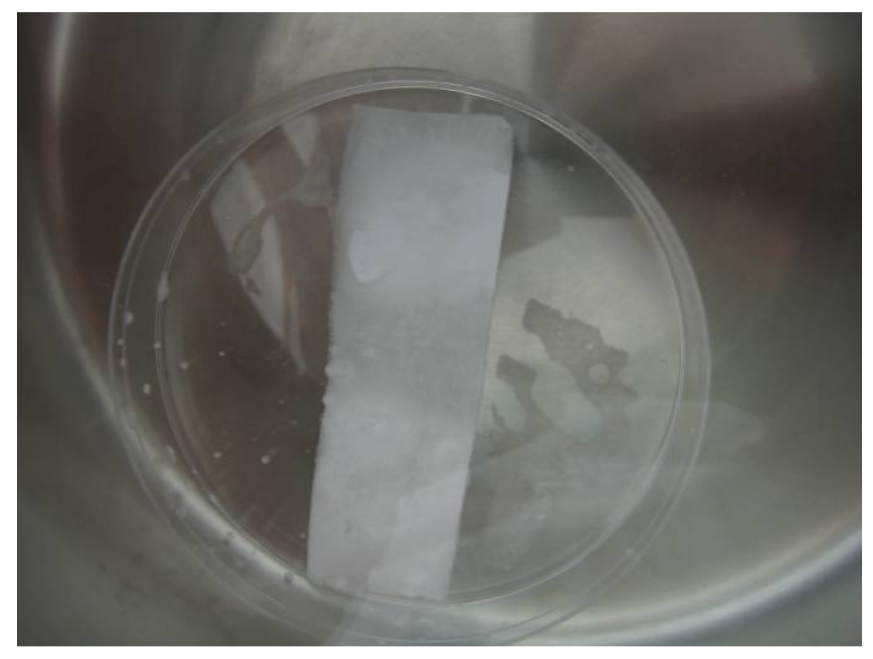

Figure 3. 3 Frozen hydrated film immediately before freeze-drying process 


\subsection{Line Width Determination Experiment}

The purpose of this experiment was to determine how selectively treated films behaved when glutaraldehyde was deposited in varying line widths. A new method of glutaraldehyde deposition was introduced in this procedure.

\subsubsection{Materials and Equipment}

Tag board of $.5 \mathrm{~mm}$ thickness was used as a template material along with paint brushes, test tubes, a water bath at 37 degrees Celsius, and materials and equipment in Treatment Protocol 2.

\subsubsection{Procedure}

The casting protocol and Treatment Protocol 2 in Section 3.1 were used for film casting and treatment. Templates were prepared by cutting lines of widths $.5 \mathrm{~mm}$ to $2.5 \mathrm{~mm}$ at increments of $.5 \mathrm{~mm}$ out of tag board. Each line was $30 \mathrm{~mm}$ long. The templates were held by hand on top of the films while brushing glutaraldehyde over the film surface. The glutaraldehyde was administered three times over the same area during a 20 minute time interval. After treatment, the films were left to dry overnight. The dry films were each placed in a test tube with 37 degree milli-Q water. The tubes were placed in a water bath at a temperature of 37 degrees $\mathrm{C}$ and monitored daily. 


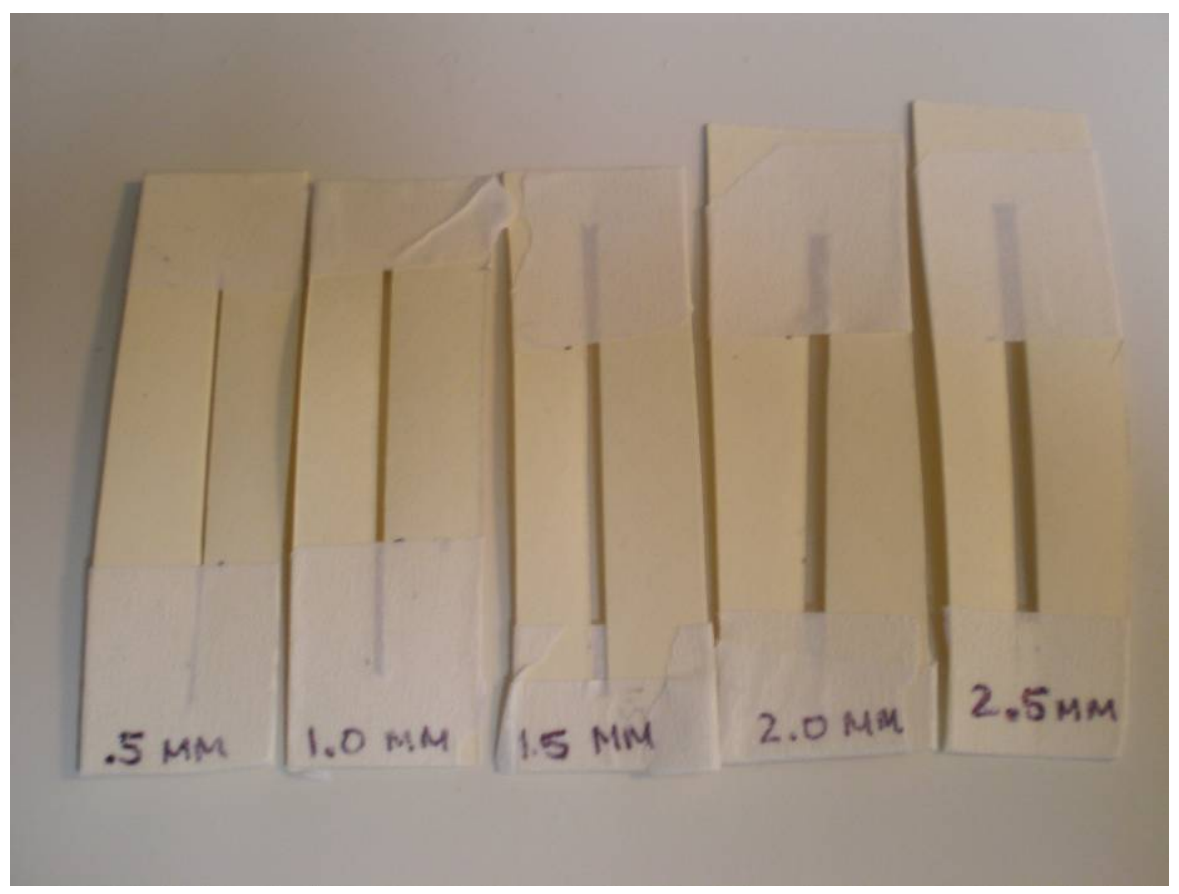

Figure 3. 4 Templates for selectively treating lines of varying widths

\subsection{Assembling a 3-D object}

The purpose of this experiment was to construct a three-dimensional object by stacking selectively treated films and observe the differences between freeze-dried and unfreeze-dried film assemblies. Two attempts were made to assemble the object with unfreeze-dried treated films.

\subsubsection{Materials and Equipment}

Plexiglass containers were constructed using an acrylic bonding agent with inner lateral dimensions of a microscope slide for the purpose of holding the films in place. Plexiglass lids were later constructed as needed in the second assembling attempt. The treatment template constructed in Solidworks was used. Figure 3.5 and Figure 3.6 show the model constructed in Solidworks and an example of the treatment template used. 


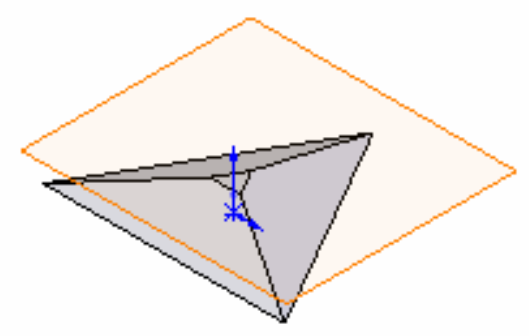

Figure 3. 5 Solidworks model used to build the treatment templates

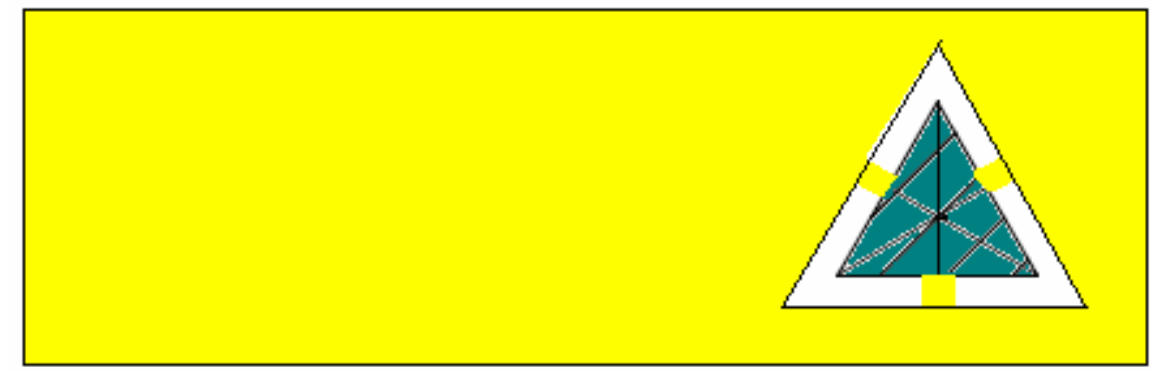

Figure 3. 6 Representation of a treated film in the first attempt at building a three-dimensional object. The yellow portion represents treated film. The white portion represents untreated film. The blue portion represents film treated with a mixture of glutaraldehyde and blue ink.

\subsubsection{Assembly Protocol 1}

This experiment was done first on unfreeze-dried films and on both types of films after protocol modification. The films were cast in the shape of a microscope slide for ease of studying the structure after the treatment. Treatment Protocol 2 was used with the treatment templates created in Solidworks. The frame and anchoring arms were treated with clear glutaraldehyde. A blue ink and glutaraldehyde mixture was used to treat the desired shape within the treatment window for the purpose of distinguishing the constructed object from the surrounding frame. After the films had dried, they were stacked into clear plexiglass containers and 37 degree C milli-Q water was added. 


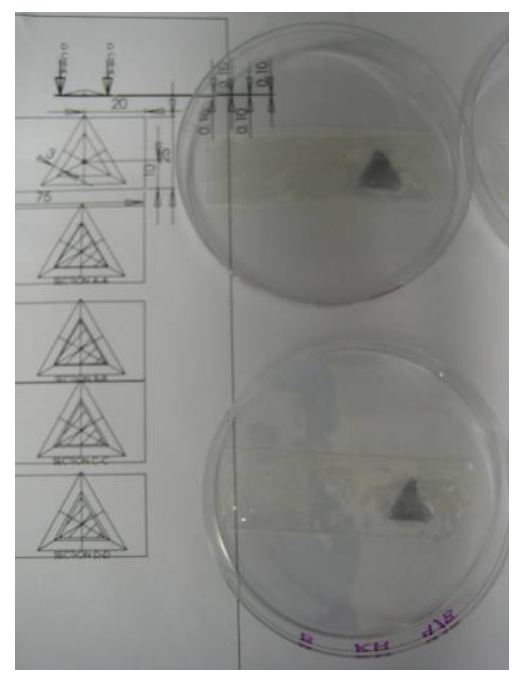

Figure 3. 7 Treatment using templates and ink before object assembly
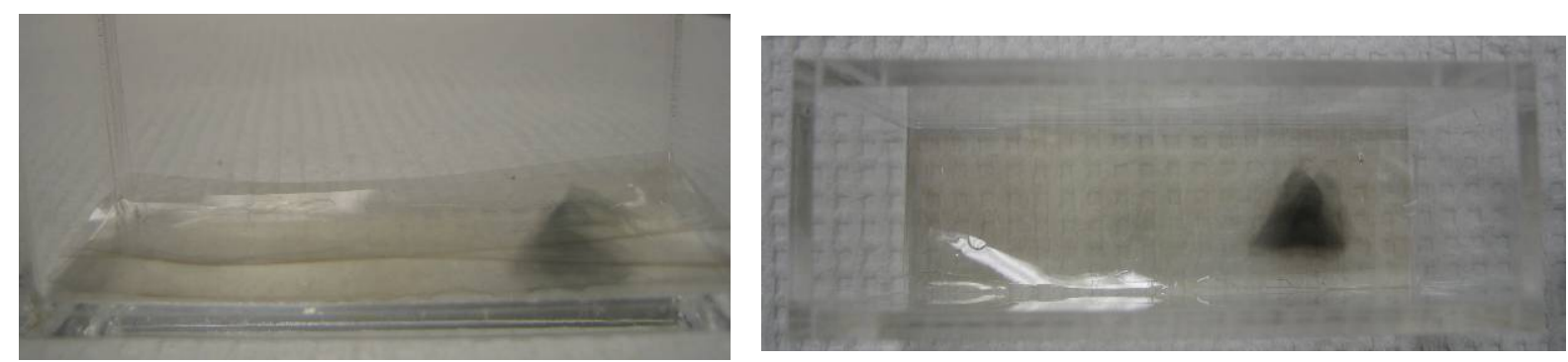

Figure 3. 8 Top and side views of films in containers

\subsubsection{Assembly Protocol 2}

This procedure included both freeze-dried films and unfreeze-dried films. The templates were redrawn with a larger untreated area to increase the film dissolution. The treatment window was also moved to the center for ease of glutaraldehyde application for the purpose of equalizing forces in solution. 


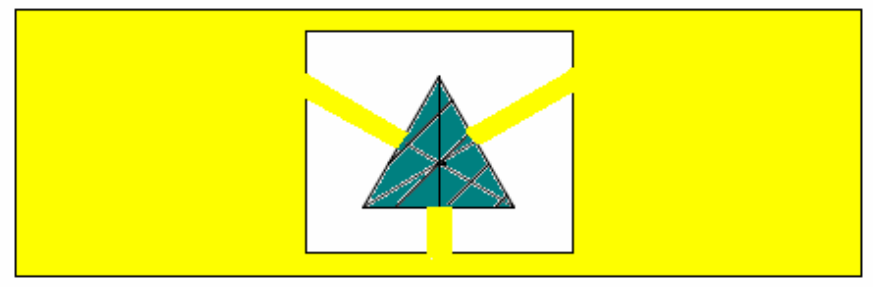

Figure 3. 9 Representation of a treated film. Yellow: treated with glutaraldehyde White: untreated film Blue: treated with a mixture of glutaraldehyde and blue ink

Plexiglass lids were constructed to restrict vertical motion of the films. The lids measured $73 \mathrm{~mm}$ in length and $24 \mathrm{~mm}$ in width. These were slightly smaller than the inner dimensions of the assembly container to allow for water to flow around the edges. Treatment Protocol 2 was used to treat the films. After the treatment was finished, half of the films were left to dry while the other half were freeze-dried. Notice that the freeze-drying step was placed at the end of this procedure rather than before treatment, as in the freeze-drying experiment in Section 3.3.

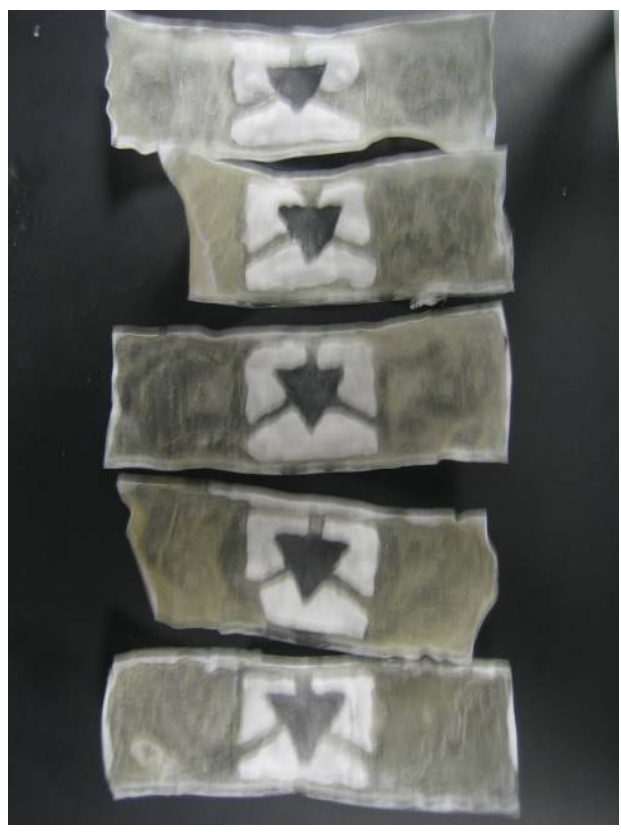

Figure 3.10 Freeze-dried films before stacking and hydrating 
When all films had dried, they were stacked and visually aligned according to the treatment windows. Irregular edges were trimmed to allow for insertion into the assembly container. The assembled films were compressed with a plexiglass lid and 37 degree Celsius milli-Q water was added. The container and lid were secured with masking tape and placed in a water bath at 37 degrees Celsius. The assembly was monitored one hour after being submerged and once a day until the untreated film dissolved.

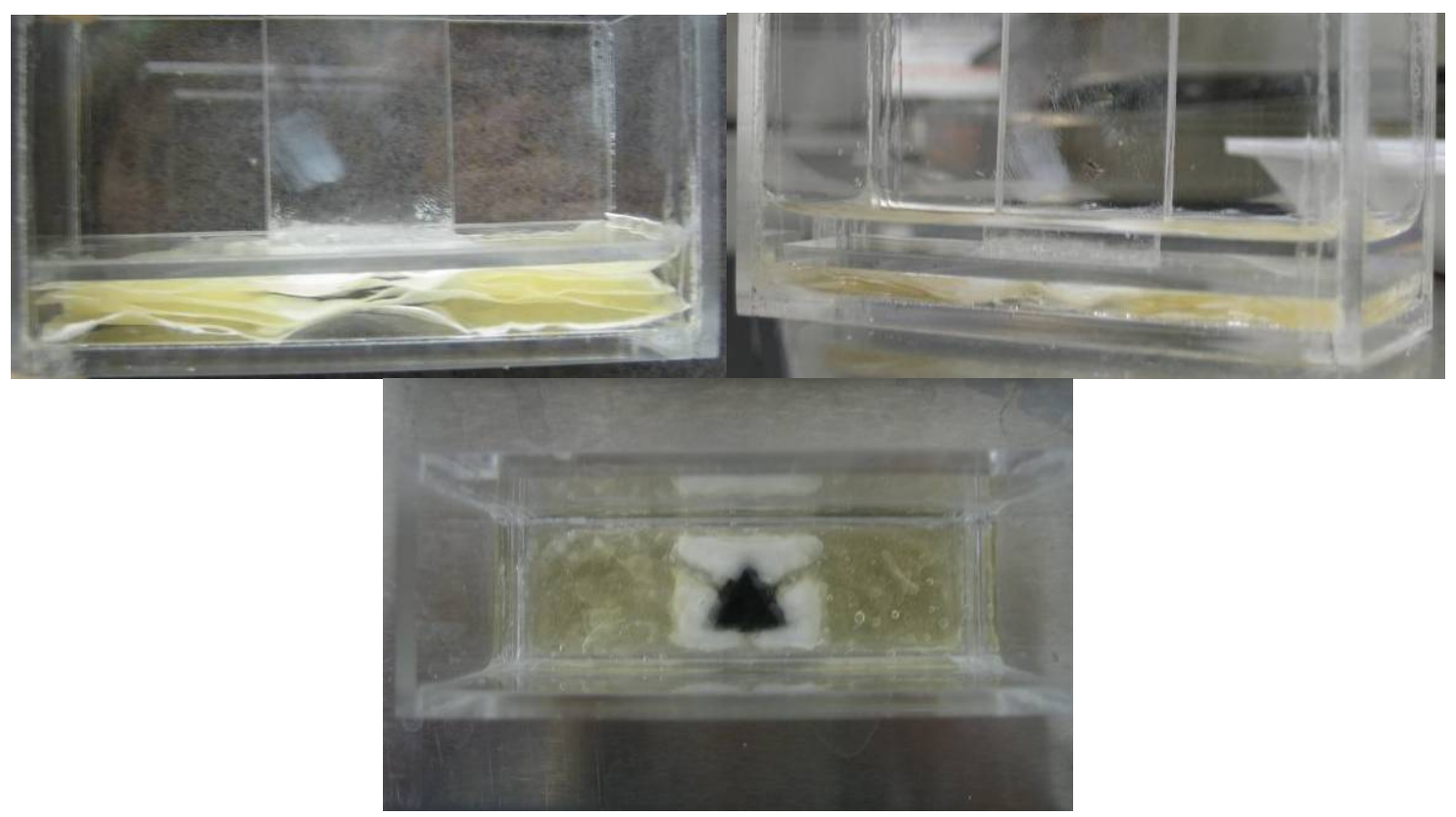

Figure 3. 11 Stacked films before hydration, immediately after hydration, and 30 minutes after hydration.

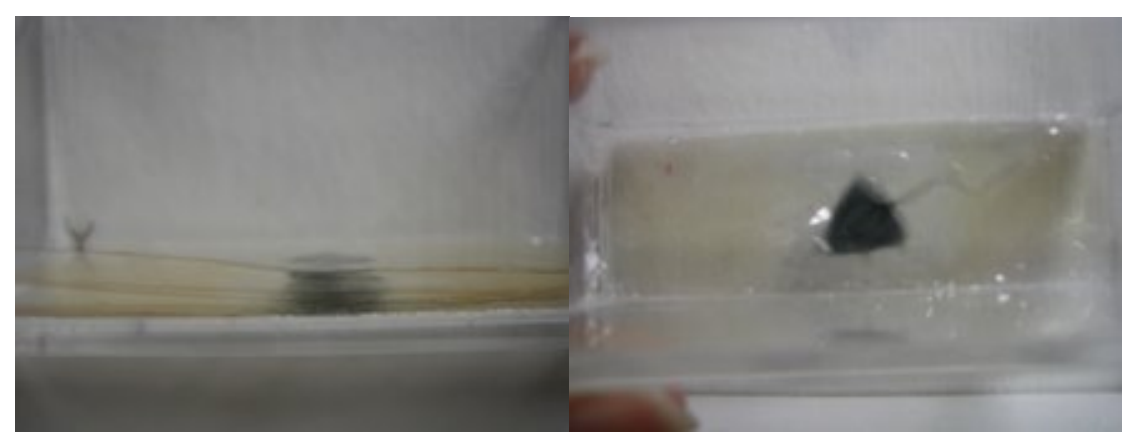

Figure 3. 12 Unfreeze-dried films before and immediately after hydration 


\section{CHAPTER 4. RESULTS AND DISCUSSION}

The following section describes the outcomes of each experiment and the implications for substrate assembly. The section concludes with suggestions for future studies of this application.

\subsection{Outline Selective Treatment Experiment Results}

\subsubsection{Results of Treatment Protocol 1}

During this treatment, most of the untreated portions of film became disfigured. This caused the treated portion to also break or disfigure on some films. Because the treated films consisted of two materials with different swelling properties, these areas were subjected to stress concentrations and could not withstand the agitation and swelling through the duration of rinsing. Thin lines were especially prone to breaking. In this experiment, the thinnest lines observed to withstand the procedure were between 2 and $3 \mathrm{~mm}$ wide. The films of $20 \%$ gelatin concentration were slightly more bendable than those of $10 \%$ concentration. However, there was no significant observed difference in film deformation.

In Figure 4.1, film a shows that different swelling ratios of each material contributes to the deformation of the entire film. It is clear the untreated film inside the circle swelled more than the treated circle and wrinkled onto itself because of excess surface area. The edges of film $\mathrm{b}$ also show a difference in material swelling resulting in wrinkled and deformed edges. The untreated portions of the films were hard and brittle at the edges and in sections that had swelled and collapsed in a wrinkled formation. Also, deformed films projected into the vertical direction, making future layering processes difficult with this process. 

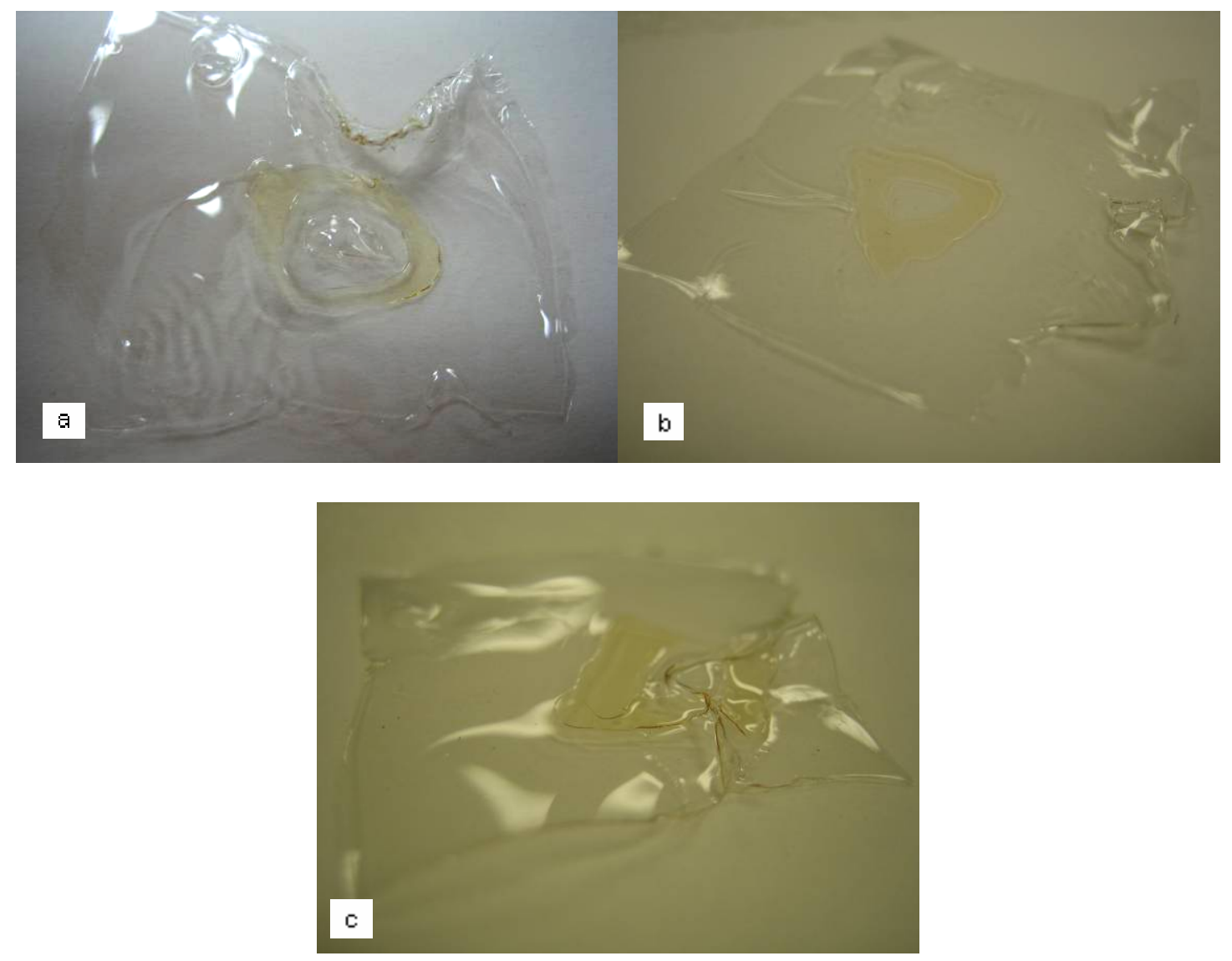

Figure 4. 1 Treated and dried films: a. 20\% gelatin film b. $10 \%$ gelatin film c. $20 \%$ gelatin film

Finally, it was verified that the treated portions of the films displayed the yellow hue described by Martin [19]. This coloration is due to the aldamine linkages between the free amino groups of protein and glutaraldehyde. The color darkened as the volume of applied glutaraldehyde increased. When treating, it was observed that the glutaraldehyde solution initially remained on the surface of the film before penetrating, exhibiting a concave formation due to surface tension of the water. Therefore, thicker lines allowed for a greater volume of glutaraldehyde to remain on the surface. In film a of Figure 4.1, the thin line appears almost clear next to the thicker portions of the shape. To create this thin line, the glutaraldehyde 
solution was dragged across the film, reducing the amount of volume on the surface of the film compared to the wider sections. This low volume may not have been sufficient to penetrate throughout the entire film. This suggests that even in thin films glutaraldehyde may not penetrate the entire film thickness unless it is allowed to sit on the surface of the film or is continuously reapplied.

\subsubsection{Results of Treatment Protocol 2}

This protocol called for the temperature and rinsing times to be reduced. Also, the mechanical stimulation of the films during rinsing was eliminated. The lack of agitation reduced swelling and the chance of tearing and wrinkling. The shapes shown on these particular films in Figure 4.2 were drawn using a metal template fabricated by Megan Barry [31]. The glutaraldehyde application suffered inaccuracies using this deposition method; however, the overall film properties were favorable for this application.
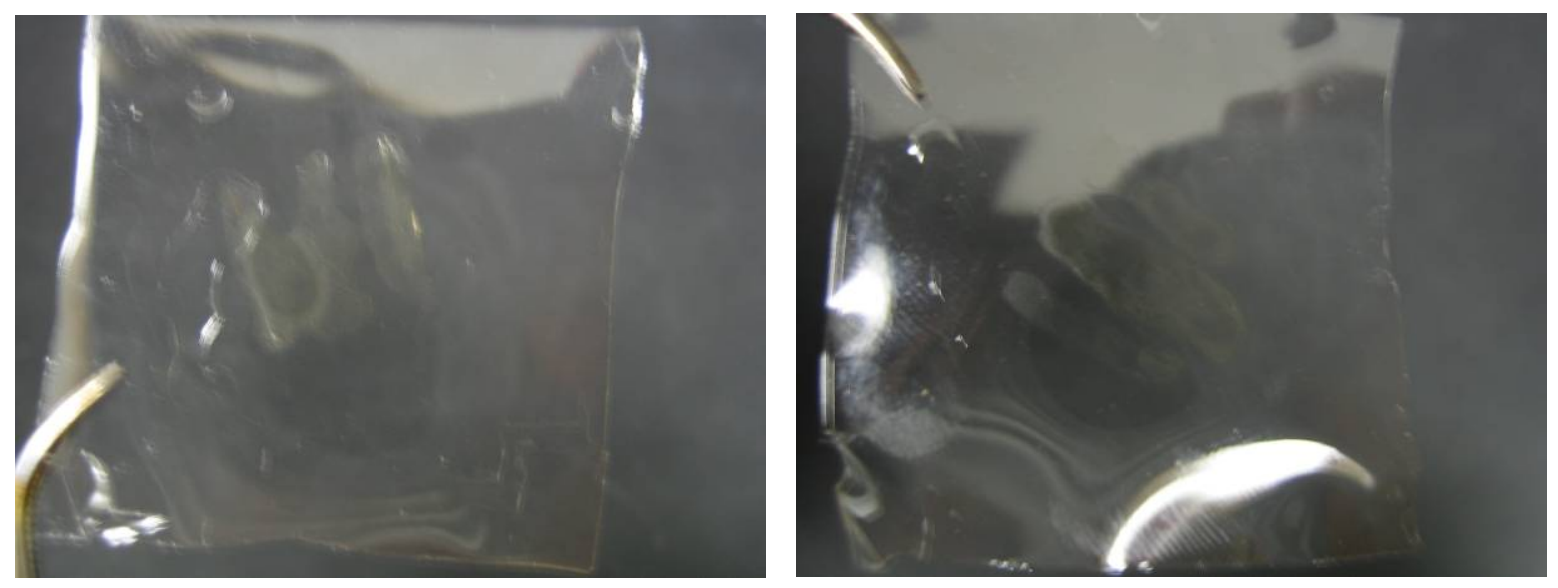

Figure 4. 2 Films produced by Treatment Protocol 2

The films produced with this revised protocol displayed many favorable characteristics. They remained flat and suffered only minor deformities. No films undergoing this treatment 
method suffered tearing or wrinkling. Because of these favorable properties and the reduced treatment time compared to Treatment Protocol 1, Treatment Protocol 2 was used for all subsequent experiments.

\subsection{Results of Film Dissolution Experiment}

The film treated with the new protocol dissolved in ten minutes. The other films took about two hours to dissolve. In all cases, the untreated gelatin dissolved, leaving only the treated portions of the films. The remaining shapes seemed to remain true to the original deposition, though no exact measurements were taken. Table 4.1 shows the films as they dissolved over time.

Table 4.1 Dissolution Test - Film Type vs. Time Submerged in Water

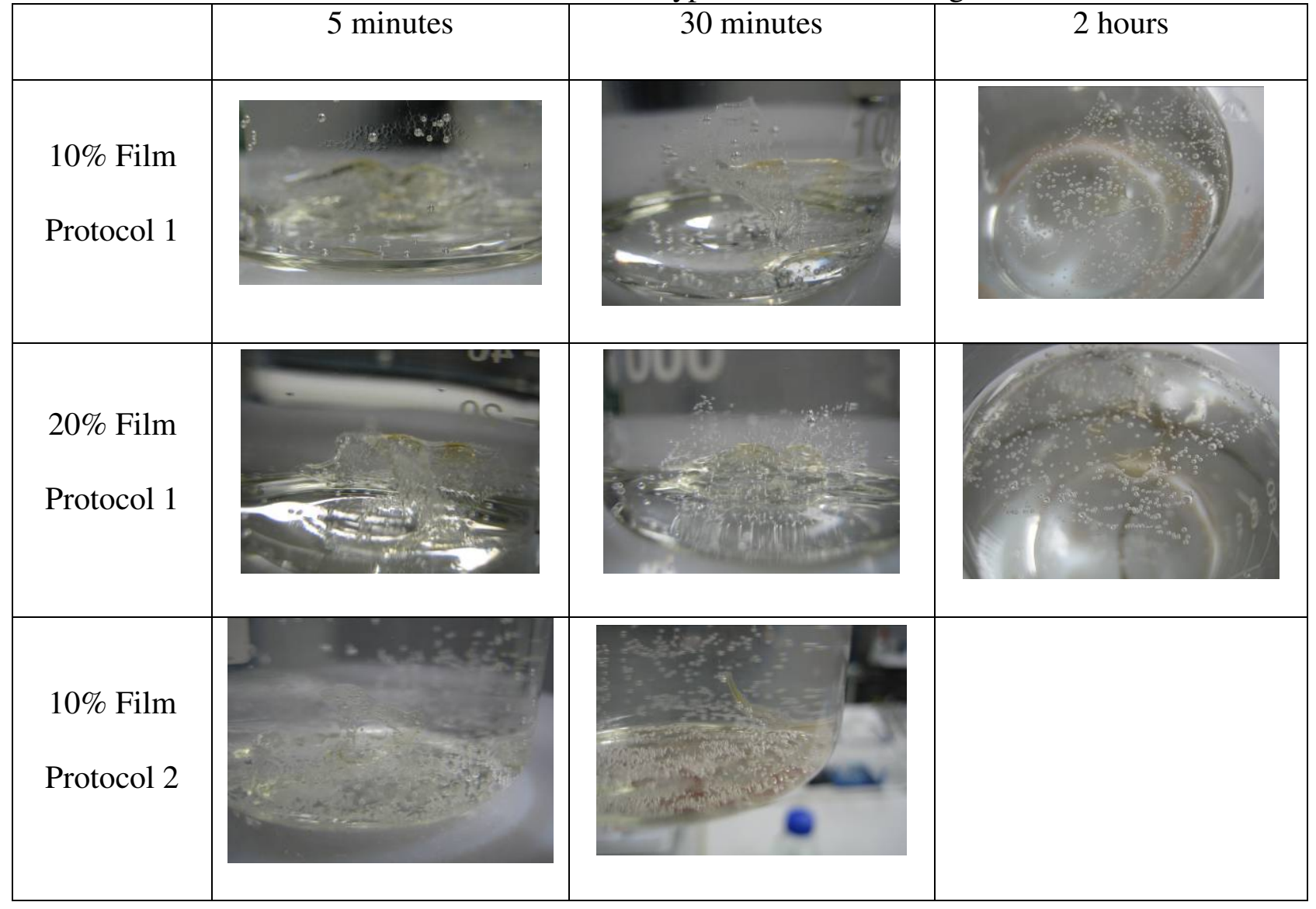


A number of factors could explain the difference of dissolution times between the films. More time had passed between completion of treatment and the dissolution experiment of films treated with Treatment Protocol 1. The films may have suffered impurities that hindered their dissolution. Also, the treatment technique may have improved in the second protocol, leaving less chance of residual glutaraldehyde and undesired cross-linking. Finally, the uniformity of the film treated according to Treatment Protocol 2 may have aided the rapid dissolution of untreated film. Variations in shape and thickness of films treated according to the first protocol may have hindered dissolution by creating dense sections that were difficult to break down. Overall, this experiment supported the use of Treatment Protocol 2 for the purpose of substrate assembly.

\subsection{Results of Treating Freeze-dried and Unfreeze-dried Films}

Results regarding the ease and effectiveness of treatment between freeze-dried and unfreeze-dried films were observed as well as that of the paint brush application method. The brushes proved effective in producing thinner lines and better accuracy compared to previous application methods. Once the glutaraldehyde was applied to the freeze-dried film, the film saturated and became transparent. The only observed difference in the ease of glutaraldehyde application resulted from the transparency of the unfreeze-dried film. The treatment template was placed beneath the film and was traced with the application brushes. The template only appeared under the freeze-dried film after it had been saturated. Otherwise, there was no observed difference in the ease of glutaraldehyde application between the freeze-dried and unfreeze-dried films. 
The films did not show any appreciable mechanical or visual differences at the end of the treatment process. It was hypothesized that the glutaraldehyde may have absorbed throughout the film thickness faster in the freeze-dried film. However, there was no sign of this in the resulting films. Both the freeze-dried and unfreeze-dried films had similar handling characteristics. It was concluded that freeze-drying before treatment was not a necessary addition to the protocol.

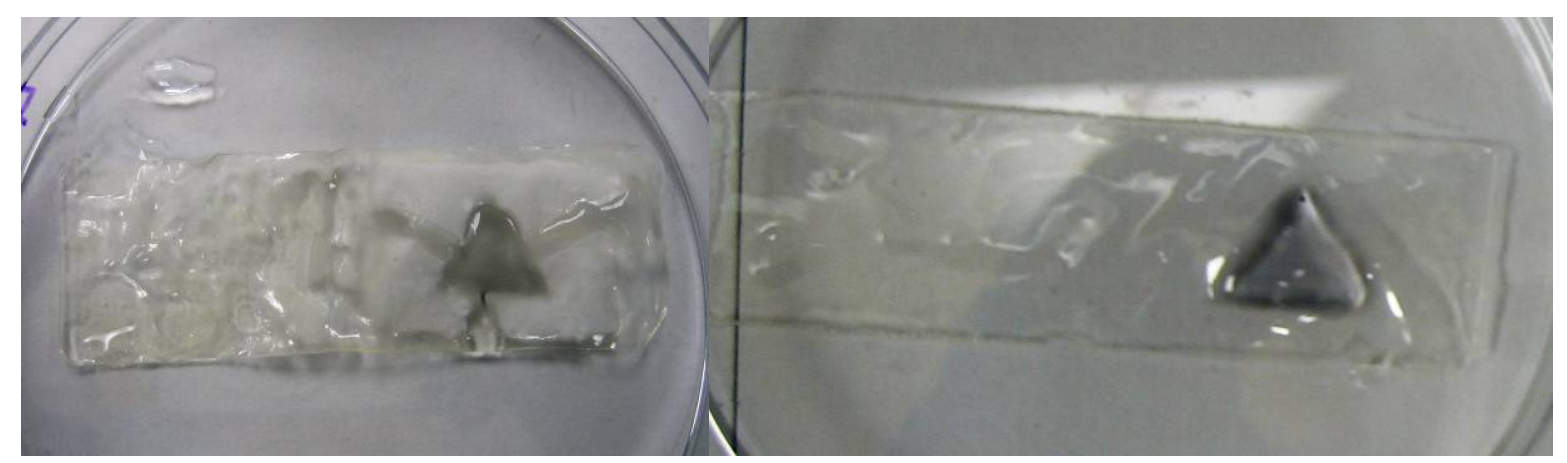

Figure 4. 3 Films immediately after glutaraldehyde treatment. Left: Freeze-dried film. Right: Unfreeze-dried film.

\subsection{Results of the Line Width Determination Experiment}

The untreated film in this experiment was found to dissolve within one day of being submerged in warm water. The expected outcome of this experiment was that the thicker films would have greater strength, stability, and definition. However, the $.5 \mathrm{~mm}$ wide line proved to last the longest with the greatest stability. Figure 4.4 shows that the thinner line did not curl or fold over on top of itself. The thicker lines curled around themselves, exhibiting weakness in their structures. One possible explanation is that the glutaraldehyde was more easily "funneled" 
onto the films by the template walls of close proximity. The wider lines allowed the spread of glutaraldehyde and less differential concentration of treatment. Also, the glutaraldehyde was applied with brush strokes and was not allowed to sit on the surface of the film. This may have reduced the amount of glutaraldehyde on the larger surface areas and resulted in weaker treated films.

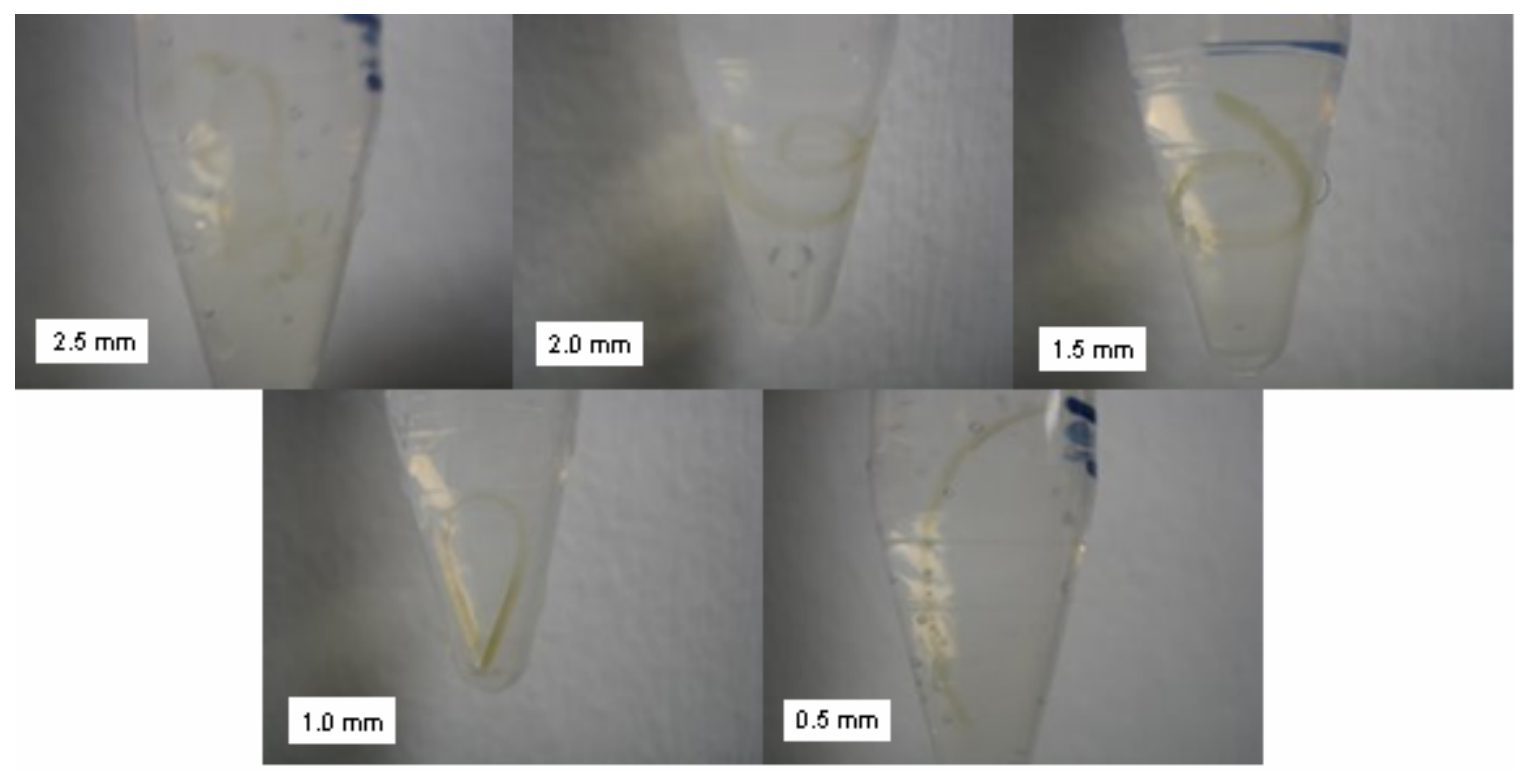

Figure 4. 4 Remaining treated lines of varying widths after dissolution of surrounding gelatin

\subsection{Results of Assembling a 3-D Object}

\subsubsection{Results of Assembly Protocol 1}

The films produced in this treatment retained their initial shape, exhibited greater strength due to the glutaraldehyde treatment of the frame, and handled easily throughout the procedure. However, the treatment window was too small to represent the dissolution of untreated film. Also, when the film container was filled with water, the films separated. It was noted that the untreated area floated and folded over the treated film. Due to these results, the protocol was 
altered to include a constraining lid that would only allow the movement of water throughout the container.

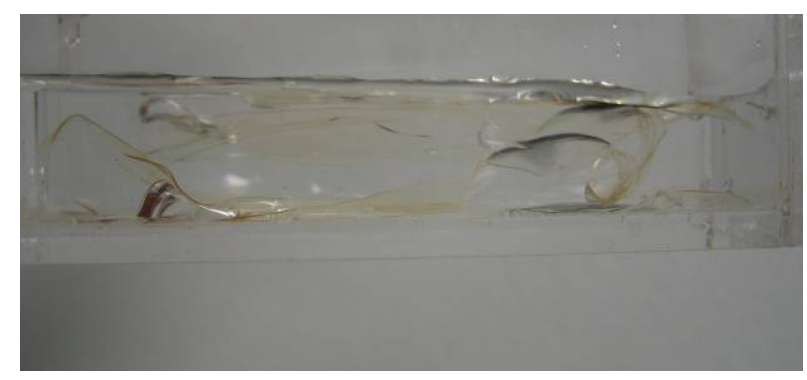

Figure 4. 5 Films immediately after adding water

\subsubsection{Results of Assembly Protocol 2}

This protocol consisted of two types of films: both types went through Treatment protocol 2. However, one type of film went through an added freeze-drying step before assembly. Differences in ease of assembly were noted. One benefit of assembling the untreated films was the elimination of the freeze-drying step. It took about four hours for the ice contained in the films to completely sublimate. Since the freeze-dryer used only had one container for this purpose, the films were freeze-dried in series. Therefore, this step added four hours per film to the entire process.

Also, the opaque and mechanically stiff properties of the freeze-dried films added to the challenge of aligning the films before assembly. Alignment depended more on the accuracy of the dimensions of the entire film, eliminating the added visual verification used to align clear films. Also, during freeze-drying the films did not remain flat, but dried in a wavy, threedimensional configuration (See Figures 3.10 and 3.11). This also caused difficulties in 
alignment and assembly. After the films were stacked, they were compressed with the lid and did not relax until water was added.

After about one week, the untreated freeze-dried film had dissolved completely leaving a well defined structure behind. However, the treated arms had slightly misaligned due to both tracing inaccuracies and alignment challenges and appeared to have increased in thickness.

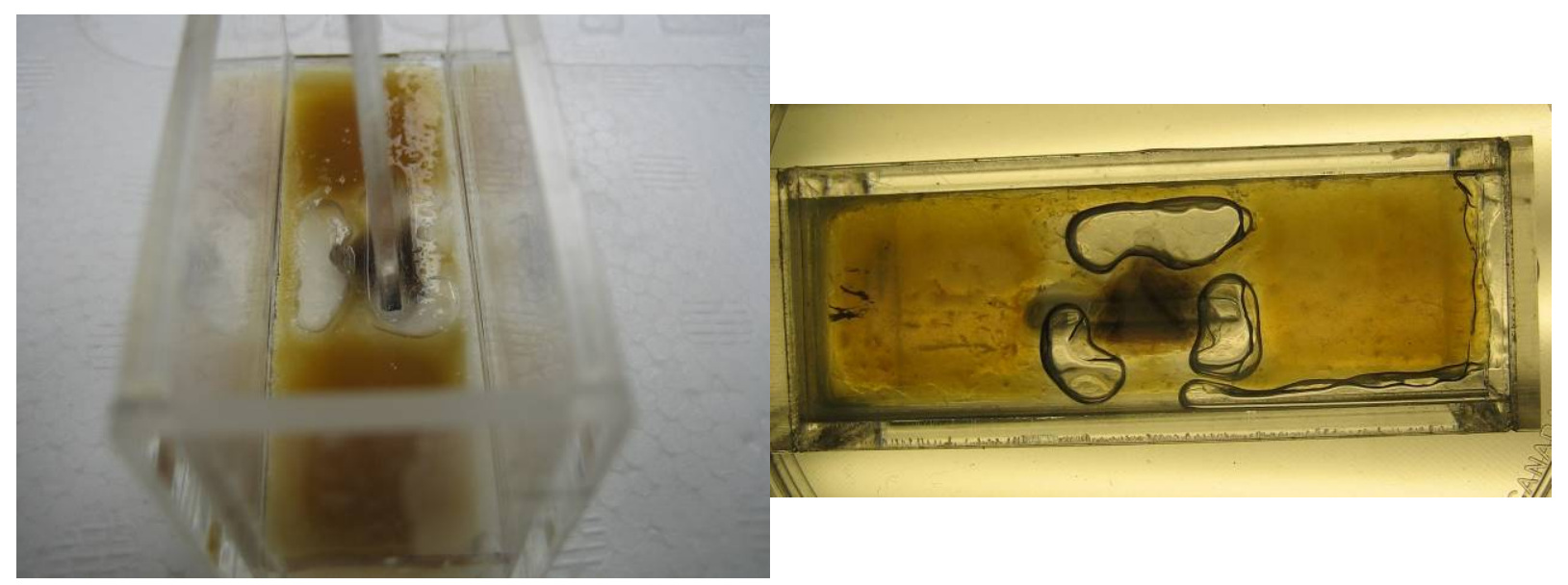

Figure 4. 6 Results of freeze-dried film assembly - top and bottom views

The unfreeze-dried films were treated and assembled easily due to their transparency and flexibility. Since they allowed for the template to be directly traced during treatment, the treatment patterns exhibited better accuracy. Also, the transparency of the films increased the alignment accuracy of the entire assembly.

One drawback of eliminating the freeze-drying step was the increase in dissolution time. As seen in Figure 4.7, the untreated film was still intact after seven days of soaking. The untreated freeze-dried film had already dissolved after this amount of time. One possible explanation is that the unfreeze-dried assembly did not provide enough room for the flow of water throughout the assembly. As a result, the untreated gelatin remained within the assembly. 


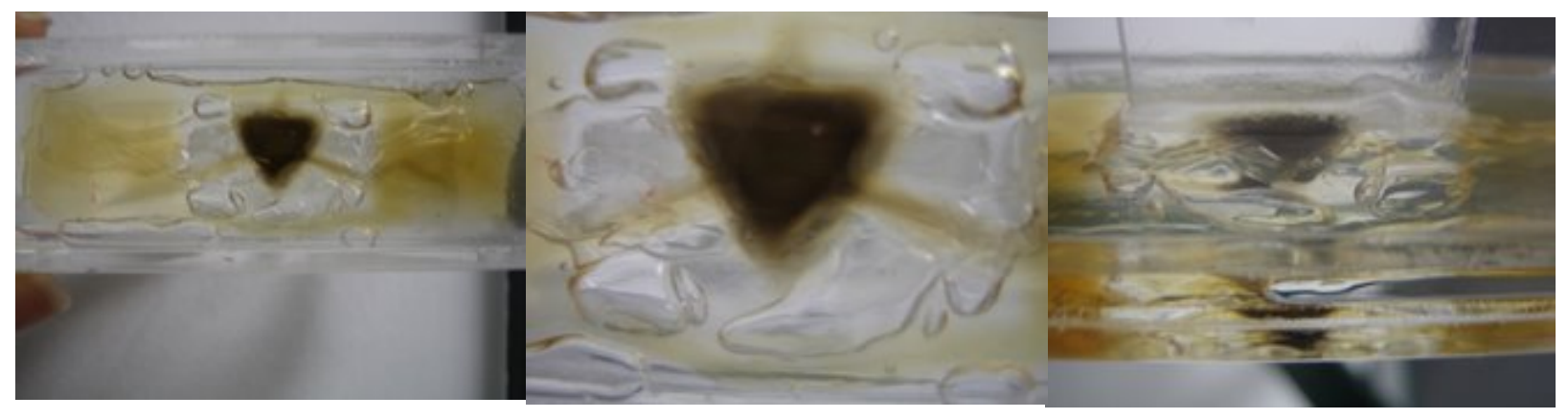

Figure 4. 7 Top view, magnified top view, and side view of the unfreeze-dried three-dimensional construct.

It was also observed that each assembly slowly degraded with time. This may indicate that this process may be useful in scaffold fabrication techniques in which substrate degradation is desired.

\subsection{Results Summary}

A few limitations and suggested practices can be drawn from these five experiments. Temperature control is necessary retain strength in the untreated films during processing as well as weaken and dissolve films during the final assembly. When saturating gelatin, lowering the water temperature will reduce film degradation and disfigurement. This will allow the gelatin to better match the properties of cross-linked film during processing. In a rapid prototyping system, stronger untreated films will help to produce a more uniform and accurate result, especially if automation is implemented.

In attempting to define line width limitations, the template method yielded a line with a width of $.5 \mathrm{~mm}$ that was more robust than the thicker lines. One explanation for this is the combination of the surface tension property of the glutaraldehyde and the short distance of the template walls. The solution may have accumulated around the walls, providing a continuous "treatment well" to be absorbed into the film. 
Freeze-drying proved to be advantageous when implemented at the end of the treatment process. The main advantage of freeze-drying was the increase in dissolution time. This open structure aided in the rapid breakdown of the untreated gelatin by reducing bonding within the gelatin and allowing the film to saturate faster. One drawback to freeze-drying is the loss of visibility when stacking the films. However, increased accuracy in the treatment process can compensate for this loss of visual confirmation when stacking.

\subsection{Future Studies}

Using the processes developed in this study, the macrostructure of a complex three dimensional shape may be obtained. Experimenting with culturing cells onto this structure is the next step in determining the feasibility of this process as a method for producing a viable substrate. It is suggested that the cells are seeded between the films during stacking. The permeability of the treated films has been investigated and indicates that an exchange of certain types and sizes of products, waste, and nutrients across a treated film is possible. Cell lines that may benefit from this three-dimensional, semi-permeable structure should be identified.

The properties of these structures may be further studied and altered. The current process provides a film that is the appropriate size for loading onto a scanning electron microscope platform. The microstructures, swelling properties, and degradation rates of the four types of films produced (depending on freeze-drying and glutaraldehyde treatment) should be observed and compared. The microstructure of the shapes may be altered by elevating the freezing temperature of the samples before freeze-drying. If desired, this alteration may provide a more porous and interconnected structure for cell proliferation and nutrient and waste exchange. Also, 
because glutaraldehyde penetration occurs slowly, the amount of glutaraldehyde deposited as well as film thickness should be closely monitored and standardized.

Resolution and repeatability may be increase by automating the process. A small ejection head should be employed for glutaraldehyde deposition. A thinning agent should be used to decrease the surface tension of the water to produce deposition droplets of smaller diameters. Studies are underway for automating this process with an inkjet printing apparatus. This automation would increase the speed and accuracy of film production. 


\section{APPENDIX A Treatment Template}

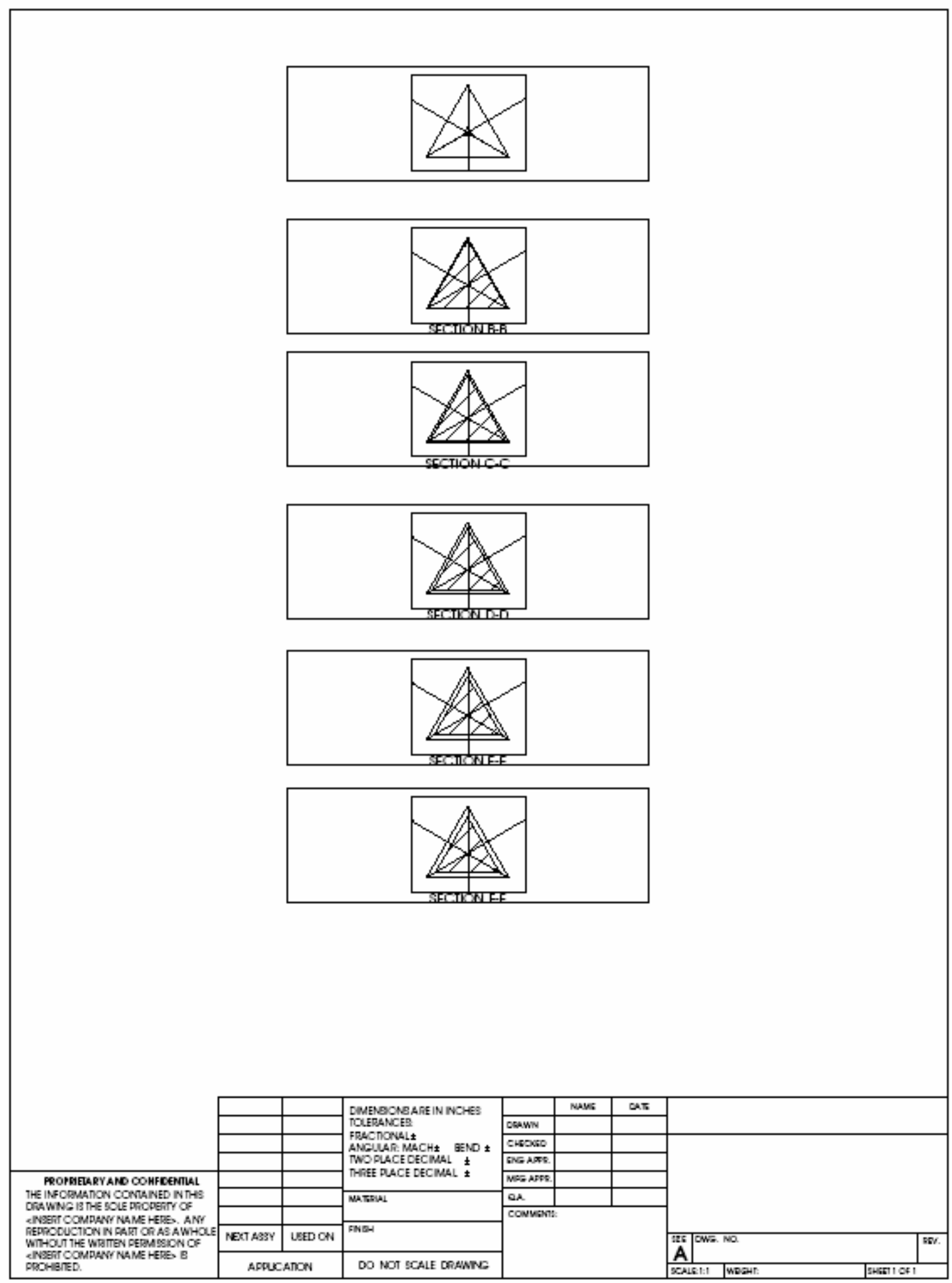




\section{REFERENCES}

1. Castro, Fidel O., Janne, Juhani, (1998). Mammary Gland Transgenesis - Therapeutic Protein Production, Springer, Georgetown, USA, 1- 5.

2. M2-Systems, "The Stereolithography Process," 2005. http://www.m2-systems.com (Accessed 10/22/07).

3. Groover, Mikell P., (2002). Fundamentals of Modern Manufacturing - Materials, Processes, and Systems, John Wiley \& Sons Inc., Hoboken, NJ, 774 - 783.

4. Castle Island Company, "Solid Ground Curing," 2006. http://home.att.net/ castleisland/sgc.htm (Accessed 9/12/07).

5. Griffith, Michelle, Lamancusa, John S., "Rapid Prototyping Technologies," Rapid Prototyping. 1998. http://www.me.psu.edu/lamancusa/me415/rpintro2.pdf (Accessed 10/22/07).

6. Weiss, Lee E., "SFF Processes," JTEC/WTEC Panel Report on Rapid Prototyping in Europe and Japan. March 1997. http://itri.loyola.edu/rp/02_02.htm (Accessed 10/22/07).

7. MIT, “Three Dimensional Printing," 2000. http://www.mit.edu/ tdp/whatis3dp.html (Accessed 10/22/07) 
8. Seitz, Hermann, Tille, Carsten, Irsen, Stephen, Bermes, Gunter, Sader, Robert, Zeilhofer, Hans-Florian, (2004). Rapid Prototyping models for surgical planning with hard and soft tissue representation. CARS International Congress Series, 1268, 567-572.

9. Gibson, I., Cheung, L.K., Chow, S.P., Cheung, W.L., Beh, S.L., Savalani, M., Lee, S.H., (2006). The use of rapid prototyping to assist medical applications, Rapid Prototyping Journal, 12, 53-58.

10. Leong, K.F., Cheah, C.M., Chua, C.K., 2002. Solid freeform fabrication of threedimensional scaffolds for engineering replacement tissues and organs, Biomaterials, 24, 2363-2378.

11. Buckley, C.T., O’Kelley, K.U., (2004), Regular scaffold fabrication techniques for investigations in tissue engineering. Topics in Bio-Mechanical Engineering, P.J. Predergast and P.E. McHugh (Eds.), 147-166.

12. Lam, C.X.F., Mo, X.M., Teoh, S.H., Hutmacher, D.W. (2002). Scaffold development using 3D printing with a starch-based polymer. Materials Science and Engineering, 20, 49-56. 
13. Darling, Andrew L., Sun, Wei, (2005). Free-Form Fabrication and Micro-CT Characterization of Poly- $\varepsilon$-Caprolactone Tissue Scaffolds, IEEE Engineering in Medicine and Biology Magazine, 78-83.

12. Saladin, Kenneth S., (2004). Anatomy and Physiology: The Unity of Form and Function, McGraw Hill Companies, Inc., New York, 211.

13. Bigi, A., Borghi, M. Cojazzi, G., Fichera, A. M., Panzavolta, S., \& Roveri, N. (2000). Structural and mechanical properties of cross-linked drawn gelatin films. Journal of Thermal Analysis and Calorimetry, 61, 451-459.

14. Lodish, Berk, Zipursky, Matsudaira, Balitmore, Darnell, (2000). Molecular Cell Biology, W. H. Freeman and Company, http://www.ncbi.nlm.nih.gov/books/bv.fcgi?call=bv.View..ShowTOC\&rid=mcb.TOC\&d epth=10 (Accessed 10/26/07).

15. Freeman, Joseph W., “Collagen Molecular Modeling,” Virginia Tech, 2007. http://www.sbes.vt.edu/freeman/research/collagen_modeling.html (Accessed 10/15/07).

16. Gross, Jerome, Nagai, Yutaka, (1965) Specific degradation of the collagen molecule by tadpole collagenolytic enzyme, Biochemistry, 1197 - 1204. 
17. Hsieh, Meng-Ju, (2007). The permeability of glutaraldehyde cross-linked gelatin films designed for mammary cell adhesion. Master thesis of California Polytechnic State University, San Luis Obispo, California.

18. Lou, Xia, Chirila, Traian V., (1999). Swelling behavior and mechanical properties of chemically cross-linked gelatin gels for biomedical use, Journal of Biomaterials Applications, 184.

19. Chaplin, Martin, (2007). Gelatin, Water Structure and Science, London South Bank University, http://www.lsbu.ac.uk/water/index2.html (Accessed 10/25/07).

20. Sigma (n.d.). G1890-Product information sheet. Retrieved August 18, 2006, from http://www.sigmaaldrich.com/sigma/product\%20information\%20sheet/g1890pis. pdf

21. Chatterji, Prabha R., (1989) Gelatin with Hydrophilic / Hydrophobic Grafts and Glutaraldehyde Cross-links, Journal of Applied Polymer Science, 2203-2212.

22. Lee, Wen-Fu, Lee, Sung-Chuan, (2004) Effect of Hydrotalcite on the Swelling and Mechanical Behaviors for the Hybrid Nanocomposite Hydrogels Based on Gelatin and Hydrotalcite, Journal of Applied Polymer Science, Vol. 100, 500-507. 
23. Natarajan, N., Shashirekha, V., Noorjahan, S.E., Rameshkumar, M., Rose, C., Sastry, T.P., (2005). Fibrin-Chitosan-Gelatin Composite Film: Preparation and Characterization, Journal of Macromolecular Science, 42, 945-953.

24. Guo, Ting, Zhao, Jianning, Chang, Jianbin, Ding, Zhi, Hong, Hao, Chen, Jiangnin, Zhang, Junfeng, (2006). Porous chitosan-gelatin scaffold containing plasmid DNA encoding transforming growth factor- $\beta 1$ for chondrocytes proliferation. Biomaterials 27 , 1095-1103.

25. Robinson, J. Paul, (2000) Preparation Techniques for Confocal Microscopy, Lecture, Purdue University Cytometry Laboratories, http://www.cyto.purdue.edu/flowcyt/educate/confocal/524Lec7/tsld001.htm (Accessed $10 / 20 / 07)$

26. Lee, J.M., Edwards, H.H.L., Pereira, C. A., Samii, S.I., (1995). Cross-linking of tissuederived biomaterials in 1-ethyl-3-(3-dimethylaminopropyl)-carbodiimide (EDC), Journal of Materials Science: Materials in Medicine, http://www.springerlink.com/content/h6n8877vw4154533/ (Accessed 10/22/07)

27. Huang-Chien, Liang, Wen-Hisung, Chang, Hsiang-Fa, Liang, Meng-Horng, Lee, HsingWen, Sung, (2004). Cross-linking structures of gelatin hydrogels cross-linked with genipin or a water-soluble carbodiimide, Journal of Applied Polymer Science, 4017 4026. 
28. Wang, Xiaohong, Yan, Yongnian, Pan, Yuqiong, Xiong, Zhuo, Liu, Haixia, Cheng, Jie, Liu, Feng, Lin, Feng, Wu, Rendong, Zhang, Renji, Lu, Qingping, (2006). Generation of Three-Dimensional Hepatocyte/Gelatin Structures with Rapid Prototyping System, Tissue Engineering 1, v. 12, no. 1

29. Cataldo, F., Ursini, O., Lilla, E., Angelini, G., (2007. Radiation-induced cross-linking of collagen gelatin into a stable hydrogel, Journal of Radioanalytical and Nuclear Chemistry, http://www.springerlink.com/content/x64tu7258540m680/ (Accessed 10/26/07).

30. Kang, Hye-Won, Tabata, Yasuhiko, Ikada, Yoshito, (1999). Fabrication of porous gelatin scaffolds for tissue engineering, Biomaterials, 1339-1344.

31. Unpublished data. California Polytechnic State University San Luis Obispo, California. Oral communication with Barry, M. (2007). 